\title{
A noncooperative approach to bankruptcy problems with an endogeneous estate
}

Citation for published version (APA):

Karagozoglu, E. (2008). A noncooperative approach to bankruptcy problems with an endogeneous estate. METEOR, Maastricht University School of Business and Economics. METEOR Research Memorandum No. 031 https://doi.org/10.26481/umamet.2008031

Document status and date:

Published: 01/01/2008

DOI:

10.26481/umamet.2008031

Document Version:

Publisher's PDF, also known as Version of record

\section{Please check the document version of this publication:}

- A submitted manuscript is the version of the article upon submission and before peer-review. There can be important differences between the submitted version and the official published version of record.

People interested in the research are advised to contact the author for the final version of the publication, or visit the DOI to the publisher's website.

- The final author version and the galley proof are versions of the publication after peer review.

- The final published version features the final layout of the paper including the volume, issue and page numbers.

Link to publication

\footnotetext{
General rights rights.

- You may freely distribute the URL identifying the publication in the public portal. please follow below link for the End User Agreement:

www.umlib.nl/taverne-license

Take down policy

If you believe that this document breaches copyright please contact us at:

repository@maastrichtuniversity.nl

providing details and we will investigate your claim.
}

Copyright and moral rights for the publications made accessible in the public portal are retained by the authors and/or other copyright owners and it is a condition of accessing publications that users recognise and abide by the legal requirements associated with these

- Users may download and print one copy of any publication from the public portal for the purpose of private study or research.

- You may not further distribute the material or use it for any profit-making activity or commercial gain

If the publication is distributed under the terms of Article $25 \mathrm{fa}$ of the Dutch Copyright Act, indicated by the "Taverne" license above, 


\section{Emin Karagozoglu}

A Noncooperative Approach to Bankruptcy Problems with an Endogenous Estate

$\mathrm{RM} / 08 / 031$

JEL code: C72, D71

\section{METE@R}

Maastricht research school of Economics of TEchnology and ORganizations

Universiteit Maastricht

Faculty of Economics and Business Administration P.O. Box 616

NL - 6200 MD Maastricht

phone : ++31433883830

fax : ++31433884873 


\title{
A Noncooperative Approach to Bankruptcy Problems with an Endogenous Estate*
}

\author{
Emin Karagozoglu ${ }^{\dagger}$ \\ Maastricht University, Department of Economics
}

September 15, 2008

\begin{abstract}
We introduce a new class of bankruptcy problems in which the value of the estate is endogenous and depends on agents' investment decisions. There are two investment alternatives: investing in a company and becoming a shareholder (risky asset) and depositing money into a bank (risk-free asset). Bankruptcy is a possible event only for the risky asset. We define a game between agents each of which aims to maximize his expected payoff by choosing an investment alternative and a company management which aims to maximize the investment in the company by choosing a bankruptcy rule. There are two types of agents in our model, who are differentiated by their incomes. We, first, consider three wellknown bankruptcy rules: the proportional rule, the constrained equal awards rule and the constrained equal losses rule. We show that there always exists a pure strategy subgame perfect Nash equilibrium, which involves the proportional rule. This result is independent of the income distribution in the economy and holds even under one-sided uncertainty on the income distribution. We also show that our results can be extended to a larger set of rules containing the Talmud rule along with other rules that belong to the TAL-family. Our results provide, at least, a partial explanation from a strategic point of view for the popular use of the proportional rule in allocating bankrupt companies' assets to shareholders.

JEL Codes: C720, D710.

Keywords: Bankruptcy Problems, Subgame Perfect Equilibrium, Proportional Rule, Constrained Equal Awards Rule, Constrained Equal Losses Rule.
\end{abstract}

${ }^{*}$ I would like to thank my advisor, Bettina Klaus, for many helpful suggestions and discussions. I am also grateful to Salvador Barberá, Kristof Bosmans, Jean-Jacques Herings, Çăgatay Kayı, Özgür Kıbrıs, Juan D. Moreno-Ternero, Hervé Moulin, Antonio Nicoló, Arno Riedl, Rene Saran, William Thomson, Peyton Young and seminar participants at $5^{\text {th }}$ Murat Sertel Student Workshop, $28^{\text {th }}$ Annual Meeting of the European Public Choice Society, $3^{\text {th }}$ Economic Design \& Collective Choice Workshop, $9^{t h}$ International Meeting of Society for Social Choice and Welfare, and Sabanci University for their comments. All remaining errors are mine.

${ }^{\dagger}$ Contact Info: Maastricht University, Department of Economics, P.O. Box 616, 6200 MD, Maastricht, The Netherlands. Phone : +31 (0) 433883925 Fax: +31 (0) 433884878 . Email: E.Karagozoglu@ALGEC.unimaas.nl. 


\section{Introduction}

\subsection{Motivation}

As early as 1985, Young argues that the incentives of agents should be incorporated into cost-sharing models. ${ }^{1}$ He summarizes the motivation of the paper in the following passage:

"The problem with these approaches (ad-hoc cost sharing mechanisms) is that they ignore the problem of motivation: why should a customer pay his allocated cost if it exceeds the benefits or the alternative cost of obtaining the service by some other means? This incentive argument is especially critical in the case of voluntary association such as a club, a public consortium, or a cartel, where the partners must first agree in the manner of splitting the costs and benefits before they can proceed with the enterprise."

Thomson (2003) also addresses the need to combine noncooperative and marketbased approaches in the following paragraph:

" An important question that we will not address is the extent to which the choice of particular division rules affects agents' incentives to make commitments that one party may in the end be unable to honor. In the context of bankruptcy, these are the incentives to loan and to borrow. In many of the other applications, the parameters of the problems to be solved also result from decisions that agents have made, and whatever rule is used at the division stage will in general have had an effect on these earlier choices. In order to handle these kinds of issues, we would need to embed division rules in a more complete model in which risk-taking, effort, and other variables chosen by agents, such as lenders, borrowers, tax payers, government agencies and others, are explicitly described, stochastic returns to economic activities are factored in, and so on."

In this paper, we introduce a new class of bankruptcy problems in which the value of the estate is endogenous and depends on agents' investment decisions that also determine their claims. Our theoretical framework incorporates important economic factors such as the income distribution, stochastic returns of risky investment projects, and return on a risk-free outside option. Our model is motivated by the following facts: (i) In real bankruptcy situations, agents might act strategically and in line with their incentives, (ii) bankruptcy might occur following an investment decision with stochastic outcomes, (iii) the choice of the bankruptcy rule and investment decisions have impacts on each other through incentives, (iv) the claims distribution might have an impact on agents' decisions if there are peer effects, and (v) many real bankruptcy situations involve payments (to shareholders, lenders, partners etc.), which are not respected by the borrower.

In our model, there are two investment alternatives: investing in a company and becoming shareholder (risky asset) or depositing money into a bank (risk-free asset). Bankruptcy is a possible event only for the risky asset. We define a game

\footnotetext{
${ }^{1}$ I would like to thank Peyton Young for referring me to this paper.
} 
between agents each of which aims to maximize his expected payoff by choosing an investment alternative and a company management which aims to maximize the investment in the company by choosing a bankruptcy rule. This setup is also in line with some recent suggestions in favor of a more liberal bankruptcy law, which would provide a menu of rules and allows companies to choose one among them (see Hart, 2000). There are two types of agents in the model, who are differentiated by their incomes. We consider three well-known bankruptcy rules: the proportional rule, the constrained equal awards rule and the constrained equal losses rule. In the game, the company chooses the bankruptcy rule and later all agents simultaneously choose whether to invest in the risky asset (i.e., the project initiated by the company) or the risk-free asset (savings account in a bank). Our results provide a noncooperative support for the proportional rule. ${ }^{2}$ In particular, we show that there always exists a pure strategy subgame perfect Nash equilibrium that involves the proportional rule. This statement is not valid for the constrained equal awards and the constrained equal losses rules. The direct implication of this result is that the proportional rule never leads to an investment in the company that is lower than the one under the constrained equal awards rule or the constrained equal losses rule; and in some cases leads to an investment in the company strictly higher than the one under two rules. Moreover, the result supporting the proportional rule is independent of the income distribution and holds even under one-sided uncertainty on the income distribution. We also extend our results to include all rules in the TAL-family (Moreno-Ternero \& Villar, 2006). This extension is interesting since the TAL-family includes some well-known rules such as the constrained equal awards rule, the constrained equal losses rule and the Talmud rule. We conduct comparative static analyses on income distribution and risk-return parameters and offer policy implications.

\subsection{Overview of the Literature}

The bankruptcy problem was first introduced formally by O'Neill (1982). It describes a situation in which there is a perfectly divisible estate to be allocated to a finite number of agents, whose claims add up to an amount larger than the estate. ${ }^{3}$ A bankruptcy problem can be represented by a claims vector and an estate. A bankruptcy rule is a function that associates a division of the estate with every bankruptcy problem. Many real life situations such as distributing a will to inheritants, liquidating the assets of a bankrupt company, rationing, taxation, and sharing the costs of a public facility can be described using parsimonious bankruptcy models.

Following the introduction of the bankruptcy problem, most research on bankruptcy approaches the problem from a normative (axiomatic) perspective and aims to compare different rules by their properties. As Thomson (2003) shows, this normative

\footnotetext{
${ }^{2}$ For additional support for the proportional rule, we refer the reader to Chun (1988), Bergantiños \& Sanches (2002), Chambers \& Thomson (2002), Ching \& Kakkar (2001), Chun \& Lee (2007), De Frutos (1999), Gächter \& Riedl (2005; 2006), Hougaard \& Østerdal (2005), Ju (2003), Ju, Miyagawa \& Sakai (2007), and Moreno-Ternero (2002; 2006a; 2006b).

${ }^{3}$ This corresponds to Chapter 7 bankruptcy in the US bankruptcy law.
} 
approach was successful in determining strong contenders, i.e., a small set of bankruptcy rules with particularly desirable properties. The most prominent rules are the proportional rule, the constrained equal awards rule, the constrained equal losses rule and the Talmud rule. The proportional rule allocates the estate proportionally with respect to claims. The constrained equal awards rule allocates the estate as equal as possible taking claims as upper bounds and similarly the constrained equal losses rule allocates the shortage of the estate in an equal way (shares bounded below by zero). The Talmud rule behaves like the constrained equal awards rule when the estate is less than half of the total claims and like the constrained equal losses rule when the estate is more than half of the total claims. These rules will also be used in our research. For an extensive survey of the axiomatic literature, the reader is referred to Moulin (2002), Thomson (2003) and Thomson (2006).

Some researchers (Yaari \& Bar-Hillel, 1984; Moulin, 2000; and Herrero, MorenoTernero \& Ponti, 2006) offer an informal characterization of different circumstances in which a particular bankruptcy rule is the most sensible one. Some examples are the use of the proportional rule in income taxation and in the allocation of bankrupt firm's assets to shareholders, the constrained equal losses rule in the provision of health services, and the constrained equal awards rule in the allocation of a bankrupt bank's assets to depositors.

Still another approach to bankruptcy problems is the game theoretical approach. There are different strands within the game theoretical approach to bankruptcy problems. Historically, the first one is the cooperative game theoretical approach. This approach transforms the bankruptcy problem to a transferable utility game or a coalitional bargaining game and studies cooperative solution concepts such as the core, the kernel etc. Aumann \& Maschler (1985), Young (1985), Curiel, Maschler \& Tijs (1987) and Dagan \& Volij (1993) are some of the papers among others, which apply cooperative game theoretical approach to analyze bankruptcy problems.

We know that incentives and strategic behavior play a significant role in bankruptcy problems in real life. Hence, a natural and a fruitful approach to bankruptcy problems is a noncooperative game theoretical one. The noncooperative game theoretical approach models the bankruptcy problem as a noncooperative game among the claimants and studies the equilibrium of the game. This approach also provides a noncooperative support for bankruptcy rules. There are only a handful of papers with this approach. O'Neill (1982), Chun (1989), Dagan, Serrano \& Volij (1997), MorenoTernero (2002), Herrero (2003), García-Jurado, González-Díaz \& Villar (2006) and Chang \& Hu (2008) are the papers, which apply noncooperative game theoretical approach to analyze bankruptcy problems. Ching \& Kakkar (2001) and Araujo \& Páscoa (2002) approach the problem from a slightly different perspective by offering market based formulations. The major motivation of all these studies is that when the authority does not have a priori preferences concerning the rule that will be implemented, it might resort to implementing a noncooperative game form (a set of rules or procedures) in which the strategic interactions of claimants determine the rule to be used in equilibrium. Depending on the strategic game form the policymaker implements, the resulting equilibrium rules may differ as the studies mentioned above 
show.

The paper most closely related to ours is Kibris \& Kibris (2008). They also analyze the investment implications of prominent bankruptcy rules. The major differences between our models can be listed as: (i) In our model, the bankruptcy rule decision is embedded in a sequential game, whereas in their paper they compare investment volumes under different bankruptcy rules, (ii) in our paper, agents invest either nothing or everything in the risky asset, whereas in their paper agents solve an optimization problem to determine this amount, (iii) in our paper there are two types of agents (an arbitrary number of them), whereas in their paper, there are two agents, and (iv) in our paper, agents are risk neutral and there is a risk-free outside option, whereas in their paper, they allow for risk aversion and there is no outside option. As a result of similarities between our models, some of our results are identical. For instance, in both papers, the proportional rule leads to more investment than the constrained equal awards rule. The major difference between our results is that in our paper, the proportional rule leads to more investment than the constrained equal losses rule, whereas in their paper, this is not always valid.

\subsection{Our Contribution}

Contributions of our paper can be listed as: (a) endogenizing the determination of the bankruptcy rule with a noncooperative procedure, (b) endogenizing the value of claims and the estate, (c) incorporating the well-known bankruptcy model into a context that involves decision-making under uncertainty and mimicks a market environment, (d) explaining a real-life phenomenon, which is the use of the proportional rule in allocating bankrupt company's assets to shareholders (e) providing a noncooperative justification for the equity theory of Selten (1978) in a relevant context and (f) providing a noncooperative framework in which the bankruptcy rule decision depends on both company's and agents' incentives. Firstly, endogenously determined bankruptcy rule, claims and estate are new in the literature. In most of the papers on bankruptcy, the analysis is based on exogenously fixed bankruptcy rule, claims and the estate. In real life, obviously agents' decisions and hence the value of claims and the estate depend on the bankruptcy rule and the bankruptcy rule choice depends in turn on agent's actions. Thus, the bankruptcy rule, claims and estate are all endogenously determined. Secondly, many real life instances that involve a bankruptcy problem also involve an investment decision under uncertainty. In our paper, we model the whole investment process at an earlier stage, i.e. before bankruptcy is realized. ${ }^{4}$ With this approach, we also incorporate factors that play important roles in real-life bankruptcy problems such as stochastic returns, risk, attitudes towards risk and income distribution into the bankruptcy problem. Our model gives, at least, a partial explanation for the popular use of the proportional rule in the liquidation process of a bankrupt company and also its use in each priority class in sequential priority rules employed by some bankruptcy laws such as the US bankruptcy law

\footnotetext{
${ }^{4}$ In fact, in our model, bankruptcy might not occur. It is the possibility of bankruptcy on which company's and agents' decisions are based.
} 
(see Chapter 11). In a slightly different literature, the equity theory of Selten (1978) emphasizes the relative value of inputs and outputs. Since changes in the value of the estate does not affect this value, the equity theory predicts the use of the proportional solution in our context. Hence, our paper provides a noncooperative justification for the equity theory in the bankruptcy context. Finally, in all bankruptcy papers with noncooperative approaches, the strategic interaction takes place among claimants, whereas the bankruptcy rule decision is influenced by both lenders' and borrower's interests in our paper. It is determined as a result of a sequential game played among the lender and borrowers. Hence, our paper is the first one that involves both lenders (potential claimants) and a borrower.

\subsection{Road Map}

The organization of the paper is as follows: We first introduce the standard bankruptcy problem and the bankruptcy rules that we employ in this paper and provide some preparatory results in Section 2. In Section 3, we introduce the strategic model of bankruptcy under uncertainty and the bankruptcy problem with an endogenous estate. In Section 4 and its subsections, we analyze the equilibria of the bankruptcy game introduced in Section 3 and present some extensions. Section 5 contains comparative static analyses on risk-return and income distribution parameters. In Section 6, we discuss our assumptions, results, and future research on the topic. Finally, Section 7 concludes.

\section{Bankruptcy Problems and Rules}

Bankruptcy is typically defined as a situation in which the total claims of claimants exceed the size of the available estate. It is sometimes also referred to as the conflicting claims problem. Formally, a bankruptcy problem is represented by a set of claimants $N=\{1,2, \ldots, n\}$, a claims vector $C=\left(c_{1}, c_{2}, \ldots, c_{n}\right)$ and $c_{i} \in \mathbb{R}_{++}$, an estate $E \in \mathbb{R}_{+}$ to be divided among the claimants and the inequality $\sum_{i \in N} c_{i}>E$. We denote the set of all such bankruptcy problems $(C, E)$ by $\mathcal{B}$.

A bankruptcy rule is a mechanism that allocates the estate to claimants given any bankruptcy problem. Formally, a bankruptcy rule $F$ is a function mapping each bankruptcy problem $(C, E) \in \mathcal{B}$ into $\mathbb{R}_{+}^{n}$ such that for all $i \in N, F_{i}(C, E) \in\left[0, c_{i}\right]$ and $\sum_{i \in N} F_{i}(C, E)=E$. Below, we define the bankruptcy rules we use in our analysis.

The proportional rule allocates the estate proportionally with respect to claims.

Definition 1 (Proportional Rule) For all $(C, E) \in \mathcal{B}$, we have $P(C, E) \equiv \lambda_{p} C$, where $\lambda_{p}$ is given by $\lambda_{p}=\left(E / \sum_{i \in N} c_{i}\right)$.

The constrained equal awards rule allocates the estate as equal as possible taking claims as upper bounds. 
Definition 2 (Constrained Equal Awards Rule) For all $(C, E) \in \mathcal{B}$, and all $j \in N$, we have $C E A_{j}(C, E) \equiv \min \left\{c_{j}, \lambda_{\text {cea }}\right\}$, where $\lambda_{\text {cea }}$ solves $\sum_{i \in N} \min \left\{c_{i}, \lambda_{\text {cea }}\right\}=$ $E$.

The constrained equal losses rule allocates the shortage of the estate (i.e., the total loss due to bankruptcy) in an equal way (shares bounded below by zero).

Definition 3 (Constrained Equal Losses Rule) For all $(C, E) \in \mathcal{B}$, and all $j \in$ $N$, we have $C E L_{j}(C, E) \equiv \max \left\{0, c_{j}-\lambda_{\text {cel }}\right\}$, where $\lambda_{\text {cel }}$ solves $\sum_{i \in N} \max \left\{0, c_{i}-\right.$ $\left.\lambda_{\text {cel }}\right\}=E$.

Example $1 N=\{1,2,3,4,5\}, C=(10,30,40,70,100)$ and $E=180$.

\begin{tabular}{||c|c|c|c|c|c||}
\hline \hline agent & 1 & 2 & 3 & 4 & 5 \\
\hline claim & 10 & 30 & 40 & 70 & 100 \\
\hline$P$ & 7.2 & 21.6 & 28.8 & 50.4 & 72 \\
\hline$C E A$ & 10 & 30 & 40 & 50 & 50 \\
\hline$C E L$ & 0 & 15 & 25 & 55 & 85 \\
\hline \hline
\end{tabular}

Note that under the constrained equal awards rule, claimants 1,2, and 3 receive strictly more than what they would receive under the proportional rule, whereas claimants 4 and 5 receive strictly less than what they would receive under the proportional rule. Loosely speaking, the constrained equal awards rule favors small claimants (i.e., it makes transfers from bigger claimants to smaller claimants). Also note that under the constrained equal losses rule, claimants 4 and 5 receive strictly more than what they would receive under the proportional rule whereas claimants 1,2 , and 3 receive strictly less than what they would receive under the proportional rule. Loosely speaking, the constrained equal losses rule favors big claimants (i.e., it makes transfers from small claimants to big claimants). Later, we will make use of these facts in our analysis.

Below we prove a lemma that formalizes the idea of inter-claimant transfers under the constrained equal awards and the constrained equal losses rules taking the proportional rule payoffs as a basis.

Lemma 1 Let $(C, E) \in \mathcal{B}$. Assume without loss of generality that $c_{1} \leq c_{2} \leq \ldots \leq c_{n}$. Then,

(i) there exists a critical level of claims, $c^{*}$ such that for all $i \in N$ with $c_{i}<c^{*}$, $C E A_{i}(C, E)>P_{i}(C, E)$ and for all $i \in N$ with $c_{i} \geq c^{*}, C E A_{i}(C, E) \leq P_{i}(C, E)$ and, (ii) there exists a critical level of claims, $\widetilde{c}$ such that for all $i \in N$ with $c_{i}<\widetilde{c}$, $C E L_{i}(C, E)<P_{i}(C, E)$ and for all $i \in N$ with $c_{i} \geq \widetilde{c}, C E L_{i}(C, E) \geq P_{i}(C, E)$.

Proof. See Appendix B.

In Lemma 1, for a bankruptcy rule $F \in\{C E A, C E L\}$ and an agent $i \in N$, we provided some results on $F_{i}(C, E)-P_{i}(C, E)$. From now on, for all $F \in\{P, C E A, C E L\}$, 
we will denote the transfer from agent $i$ to other agents (taking the proportional payoff vector as the reference point) under rule $F$ in the bankruptcy problem $(C, E)$ by $S_{i}(C, E, F)$. Hence, $S_{i}(C, E, F)=F_{i}(C, E)-P_{i}(C, E)$. The following lemma provides closed form expressions for transfers under $C E A$ and $C E L$ in the model with two types of agents (see Section 3), where agents' types refer to their claims. In the model, we denote the transfer of agent $i$ of type $t$ by $S_{t}(C, E, F)$ since, by the definitions of all bankruptcy rules $F \in\{P, C E A, C E L\}$, transfers will be equal to $S_{t}(C, E, F)$, for all agents $i$ of type $t$.

Lemma 2 Let $(C, E) \in \mathcal{B}$ and let $N_{h}\left(N_{l}\right)$ denote the non-empty set of claimants each with a claim $c_{h}\left(c_{l}\right)$, with cardinality $n_{h}\left(n_{l}\right)$. Assume that $0<c_{l}<c_{h}$. Denote the set of all claimants by $N$ with cardinality $n=n_{h}+n_{l}$. Then the following statements are valid.

(a-1) If $c_{h}>c_{l}>\lambda_{c e a}$, then

(a-1-1) $S_{l}(E, C, C E A)=\frac{n_{h} E\left[c_{h}-c_{l}\right]}{\left[n_{h}+n_{l}\right]\left[n_{h} c_{h}+n_{l} c_{l}\right]}>0$ and

(a-1-2) $S_{h}(E, C, C E A)=\frac{n_{l} E\left[c_{l}-c_{h}\right]}{\left[n_{h}+n_{l}\right]\left[n_{h} c_{h}+n_{l} c_{l}\right]}<0$.

(a-2) If $c_{h}>\lambda_{c e a} \geq c_{l}$, then

(a-2-1) $S_{l}(E, C, C E A)=\frac{c_{l}\left[n_{h} c_{h}+n_{l} c_{l}-E\right]}{\left[n_{h} c_{h}+n_{l} c_{l}\right]}>0$ and

(a-2-2) $S_{h}(E, C, C E A)=\frac{n_{l} c_{l}\left[E-n_{h} c_{h}-n_{l} c_{l}\right]}{n_{h}\left[n_{h} c_{h}+n_{l} c_{l}\right]}<0$.

(b-1) If $c_{h}>c_{l}>\lambda_{c e l}$, then

(b-1-1) $S_{l}(E, C, C E L)=\frac{n_{h}\left[c_{l}-c_{h}\right]\left[n_{h} c_{h}+n_{l} c_{l}-E\right]}{\left[n_{h}+n_{l}\right]\left[n_{h} c_{h}+n_{l} c_{l}\right]}<0$ and

(b-1-2) $S_{h}(E, C, C E A)=\frac{n_{l}\left[c_{h}-c_{l}\right]\left[n_{h} c_{h}+n_{l} c_{l}-E\right]}{\left[n_{h}+n_{l}\right]\left[n_{h} c_{h}+n_{l} c_{l}\right]}>0$.

(b-2) If $c_{h}>\lambda_{c e l} \geq c_{l}$, then

(b-2-1) $S_{l}(E, C, C E L)=-\frac{E}{n_{h} c_{h}+n_{l} c_{l}} c_{l}<0$ and

(b-2-2) $S_{h}(E, C, C E L)=\frac{n_{l}}{n_{h}} \frac{E c_{l}}{\left[n_{h} c_{h}+n_{l} c_{l}\right]}>0$.

Proof. See Appendix B.

Note that for all $F \in\{P, C E A, C E L\}, n_{h} S_{h}(C, E, F)+n_{l} S_{l}(C, E, F)=0$. Hence, the transfers are balanced. This is implied by the efficiency property embedded in the definition of a bankruptcy rule. 


\section{A Strategic Model of Bankruptcy with an Endogenous Estate}

There are $n_{h}$ agents each with income $w_{h}$ and $n_{l}$ agents each with income $w_{l}$, such that $0<w_{l}<w_{h} .{ }^{5}$ Accordingly, $N_{h}$ is the set of type $h$ agents with $\left|N_{h}\right|=n_{h}$ and $N_{l}$ is the set of type $l$ agents with $\left|N_{l}\right|=n_{l}$. We use $t$ to refer to a generic type i.e., $t \in\{l, h\}$. Therefore, for all agents $i \in N_{l} \cup N_{h}$, individual income $w_{i} \in\left\{w_{l}, w_{h}\right\}$. Both types of agents are risk-neutral. Hence, each agent wants to choose the investment alternative that brings the maximum expected return. There are two investment alternatives: agents either invest in a company and become shareholders or deposit their money into a bank. The company has a risky investment project and depositing money into a bank brings a risk-free return. The state space for the outcome of the risky investment project is $\Omega=\{s, f\}$ where $s$ represents success and $f$ represents failure. Hence, the outcome of the project is a random variable $\omega$. With probability $\operatorname{Pr}(\omega=s)=\pi_{s}<1$, the investment project is successful and brings a payoff of $0<r_{s} \leq 1$ to the company; with probability $\operatorname{Pr}(\omega=f)=1-\pi_{s}$, the investment project fails and brings a payoff of $r_{f}<r_{s} \leq 1$ to the company. The company promises to pay $r$ to the depositors, which satisfies $0 \leq r_{f}<r \leq r_{s} \leq 1$. $^{6}$ However, if the project fails it cannot honor all claims since $r_{f}<r$. On the other hand, the savings account at the bank pays a constant return $\bar{r}$. We eliminate two cases that would lead to trivial results: $r_{f}>\bar{r}$ and $\bar{r}>r$. If $r_{f}>\bar{r}$ was the case, then no agent would prefer to deposit their money to the bank and if $\bar{r}>r$ was the case, then no agent would prefer to invest in the company. Hence, to make the problem interesting, we assume that $r_{f}<\bar{r}<r$. Thus, the risky asset offers a higher return in case of success, but a lower return in case of failure (bankruptcy). ${ }^{7}$ Having introduced the necessary parameters, now we define the particular class of bankruptcy problems we analyze.

Definition $4 A$ bankruptcy problem with an endogenous estate is a pair $(C, E)$, where $C$ is a claims vector with entries $c_{i}=(1+r) w_{i}$ for all $i \in N$ and $E=$ $\left(1+r_{f}\right) \sum_{i \in N} w_{i}$ is the estate. The class of bankruptcy problems with an endogenous estate is denoted by $\widetilde{\mathcal{B}}$.

Our analysis will focus on the class of bankruptcy problems with an endogenous estate, unless otherwise stated. All parameters mentioned above are common knowledge. Obviously, bankruptcy is a possible event only for the first investment alternative.

\footnotetext{
${ }^{5}$ In fact, what we mean by $w_{t}$ is the part of the income that is reserved for investment by a type $t$ agent.

${ }^{6} r$ is not determined as a result of an optimization problem. However, accepting $r$ as given is a frequently made assumption in the decisionmaking under uncertainty and the corporate finance literature. Hence, we take $r$ as given.

${ }^{7}$ We will use investing in the risky asset versus investing in the company interchangeably; and investing in the risk-free asset versus depositing money into the bank interchangeably.
} 
The management of the company, $m$, is an important player ${ }^{8}$. It chooses a bankruptcy rule $F$, which will be implemented in case of bankruptcy. The management's objective is to maximize the investment in the company. Note that, given $r, r_{s}, r_{f}$, $\bar{r}$ and $\pi_{s}$, maximizing the investment volume is identical to maximizing the profit. The bankruptcy rule chosen affects agents' investment decisions since it affects their return in case of bankruptcy. Hence, the company takes into account the possible actions of agents while choosing the bankruptcy rule. As mentioned before, we use the proportional rule, the constrained equal awards rule, and the constrained equal losses rule as benchmarks. Accordingly, the company's strategy space is denoted by $\psi_{m}=\{P, C E A, C E L\}$. The company's decision is observed by all agents. Hence, each decision of the company starts a proper subgame to be played by agents. We denote these three subgames by $\Gamma^{P}, \Gamma^{C E A}$, and $\Gamma^{C E L}$.

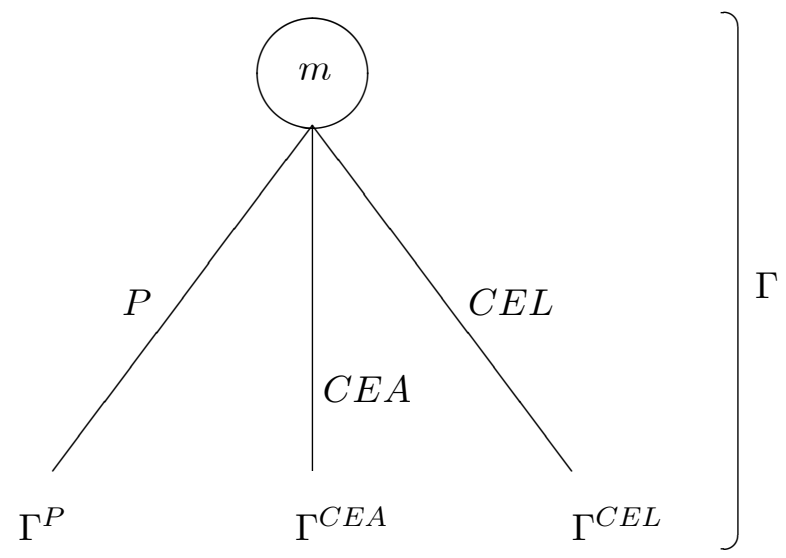

Graph 1: Game Tree

Knowing which bankruptcy rule $F$ is chosen by $m$, in the subgame $\Gamma^{F}$, all agents $i \in N_{l} \cup N_{h}$ choose whether to invest their money in the risky asset (i.e., playing in) or to invest in the risk-free, outside asset (i.e., playing out). This decision is made by all agents simultaneously. For all $i \in N_{l} \cup N_{h}$, we denote agent $i$ 's actions by $a_{i} \in\{$ in, out $\}$ and the actions taken by agent $i$ under rule $F \in\{P, C E A, C E L\}$ as $a_{i, F}$. We describe what each agent $i$ would do in each subgame $\Gamma^{F}$ by agent $i$ 's strategy, which is denoted by $s_{i} \in \psi_{i}$. Agent $i$ 's strategy space, $\psi_{i}$, can be written as

$$
\psi_{i}=\left\{\left(a_{i, P}, a_{i, C E A}, a_{i, C E L}\right) \mid F \in\{P, C E A, C E L\} \text { and } a_{i, F} \in\{\text { in }, \text { out }\}\right\} .
$$

The company's payoff function is linear and we denote it as $V_{m}(F)=\sum_{t \in\{l, h\}} n_{t, i n} w_{t}$, where $n_{t, i n}$ stands for the number of type $t$ agents who play in in the subgame $\Gamma^{F}$. Therefore, we can write the company's objective to maximize the investment as

\footnotetext{
${ }^{8}$ From now on, $m$ denotes the company.
} 


$$
\max _{F \in\{P, C E A, C E L\}} V_{m}(F) .
$$

Note that once $r$ and $r_{f}$ are fixed, the estate $E$ and the claims vector $C$ are both determined by agents' actions. Since agent $i$ 's payoff under bankruptcy is determined by $F, n_{h, i n}$ and $n_{l, i n}$, when writing agents' payoffs under bankruptcy, we employ the notation, $V_{i, i n}\left(F, n_{h, i n}, n_{l, i n}\right)$. Similarly, agent $i$ 's transfer under rule $F$ can be written as $S_{i}\left(F, n_{h, i n}, n_{l, i n}\right)$.

Now, given agent $i$ 's action in $\Gamma^{F}$, the payoff of agent $i \in N_{l} \cup N_{h}$ can be written as

$$
V_{i}\left(F, a_{i, F}\right)=\left\{\begin{aligned}
V_{i, \text { out }}= & (1+\bar{r}) w_{i}, \text { if } a_{i, F}=\text { out } \\
V_{i, \text { in }}^{e}\left(F, n_{h, \text { in }}, n_{l, \text { in }}\right)= & \pi_{s}(1+r) w_{i}+\left(1-\pi_{s}\right)\left[V_{i, \text { in }}\left(P, n_{h, \text { in }}, n_{l, i n}\right)\right. \\
& \left.+S_{i}\left(F, n_{h, \text { in }}, n_{l, \text { in }}\right)\right], \text { if } a_{i, F}=\text { in }
\end{aligned}\right.
$$

where the superscript $e$ refers to the expected value. ${ }^{9}$ Notice that the first part of $V_{i, i n}^{e}\left(F, n_{h, i n}, n_{l, i n}\right)$ is agent $i$ 's payoff in case of successful completion of the project and the second part is his payoff in case of bankruptcy. Also note that for the proportional rule, $S_{i}\left(P, n_{h, i n}, n_{l, i n}\right)=0$.

The following lemma enables us to simplify the notation $V_{j, \text { in }}\left(P, n_{h, i n}, n_{l, i n}\right)$, since it shows that the payoff each agent receive under $P$ is independent from other agents' types, actions etc. Consequently, we can write the payoff of agent $j$ under the proportional rule as $P_{j}$.

Lemma 3 Assume that for all $j \in N_{l} \cup N_{h}$, the claims structure is $c_{j}=(1+r) w_{j}$ and the estate is $E=\left(1+r_{f}\right) \sum_{i \in N_{l} \cup N_{h}} w_{i}$. Then $V_{j, i n}\left(P, n_{h, i n}, n_{l, i n}\right) \equiv P_{j}=\left(1+r_{f}\right) w_{j}$.

Proof. See Appendix B.

The result above is valid for any finite number of types. By Lemma 3, if agent $i$ is of type $t$, then $V_{i, i n}\left(P, n_{h, i n}, n_{l, i n}\right)=\left(1+r_{f}\right) w_{t}$. We rewrite agent $i$ 's expected payoff under $P$ as

$$
P_{i}^{e}=\pi_{s}(1+r) w_{i}+\left(1-\pi_{s}\right) P_{i} .
$$

Using the expected payoff under the proportional rule, we can rewrite agent $i$ 's expected payoff under $C E A$ as

$$
\begin{aligned}
V_{i, i n}^{e}\left(C E A, n_{h, i n}, n_{l, i n}\right) & =\pi_{s}(1+r) w_{i}+\left(1-\pi_{s}\right)\left[P_{i}+S_{i}\left(C E A, n_{h, i n}, n_{l, i n}\right)\right] \\
& =P_{i}^{e}+\left(1-\pi_{s}\right) S_{i}\left(C E A, n_{h, i n}, n_{l, i n}\right),
\end{aligned}
$$

where $P_{i}^{e}$ stands for the expected payoff that agent $i$ would get under the proportional rule and $S_{i}$ is the transfer that agent $i$ makes under $C E A$. Similarly, under $C E L$, the expected payoff of agent $i$ can be rewritten as

\footnotetext{
${ }^{9}$ As one can see in (3), in defining agents' payoffs from playing in in case of bankruptcy, we use the proportional rule payoff as a benchmark.
} 


$$
\begin{aligned}
V_{i, i n}^{e}\left(C E L, n_{h, i n}, n_{l, i n}\right) & =\pi_{s}(1+r) w_{i}+\left(1-\pi_{s}\right)\left[P_{i}+S_{i}\left(C E L, n_{h, i n}, n_{l, i n}\right)\right] \\
& =P_{i}^{e}+\left(1-\pi_{s}\right) S_{i}\left(C E L, n_{h, i n}, n_{l, i n}\right)
\end{aligned}
$$

where $S_{i}$ is the transfer that agent $i$ makes under $C E L$.

Table 1 below, together with the sequence of actions described before and summarized in Graph 1 define the sequential game $\Gamma$ with three proper subgames $\Gamma^{P}, \Gamma^{C E A}$, and $\Gamma^{C E L}$ :

\begin{tabular}{||c|c||}
\hline \hline Players & $\{m\} \cup N_{l} \cup N_{h}$ \\
\hline Strategies & $\psi_{m} \times \prod_{i \in N_{l} \cup N_{h}} \psi_{i}$ \\
\hline Payoffs & $\left(V_{m}(F),\left(V_{i, s_{i}}\left(F, s_{-i}\right)\right), i \in N_{l} \cup N_{h}\right.$ \\
\hline \hline
\end{tabular}

Table 1: Sequential Game $\Gamma$

where $s_{-i}$ denotes all agents' strategies except agent $i$. We look for pure strategy equilibria of this game. Since we want to capture sequential rationality, the equilibrium concept that we employ is that of a Subgame Perfect Nash Equilibrium.

\section{Analysis of Equilibrium Decisions}

Now, we analyze the equilibria of the game defined above. We start the analysis in a backward induction fashion with the subgames $\Gamma^{F} \in\left\{\Gamma^{P}, \Gamma^{C E A}, \Gamma^{C E L}\right\}$ played among all agents $i \in N_{l} \cup N_{h}$. Therefore, in the following, when we use the term "equilibrium", it refers to the agents' equilibrium actions in the corresponding subgame. After analyzing agents' behavior in each subgame, we analyze the company's action in equilibrium. This is followed by the description of all equilibria of the game along with the resulting investment in the company.

Before the analysis of agents' investment decisions in equilibrium, we prove some preparatory lemmas and corollaries. The following corollary provides values of $c^{*}$ and $\widetilde{c}$ in the class of bankruptcy problems with endogenous estates. Since the following result provides closed form expressions for $c^{*}$ and $\widetilde{c}$ in this class, it is a corollary of Lemma 1. Recall that for all $i \in N, w_{i} \in\left\{w_{l}, w_{h}\right\}$, where $0<w_{l}<w_{h}$ and $|N|=n=n_{h}+n_{l}$.

Corollary 1 Let $(C, E) \in \widetilde{\mathcal{B}}$. Then,

(i) $c^{*}=\frac{n_{h, i n}}{n_{i n}-n_{l, i n}}(1+r) w_{h}-\frac{n_{l, i n}}{n_{i n}-n_{l, i n}} \frac{(1+r)\left(r-r_{f}\right)}{\left(1+r_{f}\right)} w_{l}$ and

(ii) $\widetilde{c}=\frac{n_{h, i n}}{n_{i n}-n_{l, i n}}(1+r) w_{h}-\frac{n_{l, i n}}{n_{i n}-n_{l, i n}} \frac{(1+r)\left(1+r_{f}\right)}{\left(r-r_{f}\right)} w_{l}$. 
Proof. Recall formulas (12) and (13) from Lemma 1. If we plug into (12) and (13)

$$
\begin{aligned}
\left(1+r_{f}\right)\left(n_{h, i n} w_{h}+n_{l, i n} w_{l}\right) & \text { for } E, \\
(1+r) w_{i} & \text { for } c_{i}, \text { and } \\
(1+r)\left(n_{h, i n} w_{h}+n_{l, i n} w_{l}\right) & \text { for } \quad \sum_{i \in N} c_{i}
\end{aligned}
$$

then we obtain the equations in (i) and (ii).

The following corollary of Lemma 2 derives closed form functions of transfers under $C E A$ and $C E L$.

Corollary 2 Let $(C, E) \in \widetilde{\mathcal{B}}$.

(a-1) If $c_{h}>c_{l}>\lambda_{c e a}$, then

(a-1-1) $S_{l}\left(C E A, n_{h, i n}, n_{l, i n}\right)=\frac{n_{h, i n}}{n_{h, \text { in }}+n_{l, i n}}\left[\left(1+r_{f}\right)\left(w_{h}-w_{l}\right)\right] \geq 0$ and

(a-1-2) $S_{h}\left(C E A, n_{h, i n}, n_{l, i n}\right)=\frac{n_{l, i n}}{n_{h, \text { in }}+n_{l, i n}}\left[\left(1+r_{f}\right)\left(w_{l}-w_{h}\right)\right] \leq 0$.

(a-2) If $c_{h}>\lambda_{\text {cea }} \geq c_{l}$, then

(a-2-1) $S_{l}\left(C E A, n_{h, i n}, n_{l, i n}\right)=\left(r-r_{f}\right) w_{l}>0$ and

(a-2-2) $S_{h}\left(C E A, n_{h, i n}, n_{l, i n}\right)=\frac{n_{l, i n}}{n_{h, \text { in }}}\left(r_{f}-r\right) w_{l} \leq 0$.

(b-1) If $c_{h}>c_{l}>\lambda_{c e l}$, then

(b-1-1) $S_{l}\left(C E L, n_{h, i n}, n_{l, i n}\right)=\frac{n_{h, i n}}{n_{h, \text { in }}+n_{l, i n}}\left[\left(r-r_{f}\right)\left(w_{l}-w_{h}\right)\right] \leq 0$ and

(b-1-2) $S_{h}\left(C E L, n_{h, i n}, n_{l, i n}\right)=\frac{n_{l, i n}}{n_{h, \text { in }}+n_{l, i n}}\left[\left(r-r_{f}\right)\left(w_{h}-w_{l}\right)\right] \geq 0$.

(b-2) If $c_{h}>\lambda_{c e l} \geq c_{l}$, then

(b-2-1) $S_{l}\left(C E L, n_{h, i n}, n_{l, i n}\right)=-\left(1+r_{f}\right) w_{l}<0$ and

(b-2-2) $S_{h}\left(C E L, n_{h, i n}, n_{l, i n}\right)=\frac{n_{l, i n}}{n_{h, i n}}\left(1+r_{f}\right) w_{l} \geq 0$.

Proof. Recall the formulas we derived in Lemma 2. If we plug into those formulas for all $t \in\{l, h\}$,

$$
\begin{aligned}
(1+r) w_{t} & \text { for } c_{t}, \\
n_{t, i n} & \text { for } n_{t}, \text { and } \\
\left(1+r_{f}\right)\left[n_{l, i n} w_{l}+n_{h, i n} w_{h}\right] & \text { for } E,
\end{aligned}
$$

then we obtain the result.

The following lemma shows that $c^{*}$ and $\widetilde{c}$ are always between $c_{l}$ and $c_{h}$.

Lemma 4 Let $(C, E) \in \widetilde{\mathcal{B}}$. Assume that $n_{h, i n}>0$ and $n_{l, i n}>0$. Then, for all $n_{h, i n}$ and $n_{l, i n}$, (i) $c_{l} \leq c^{*} \leq c_{h}$ and (ii) $c_{l} \leq \widetilde{c} \leq c_{h}$. 
Proof. See Appendix B.

Since if one type is making transfers the other type should be receiving transfers in the case with two types, the result mentioned in Lemma 4 is intuitive. This result is required for the comparative static analyses we conduct in the following lemma. It ensures that when the number of type $t \in\{l, h\}$ agents changes, the identity of the type making transfers and the identity of the type receiving transfers stays the same.

Lemma 5 Let $(C, E) \in \widetilde{\mathcal{B}}$. Then,

(i) an increase in $n_{l, i n}$ weakly decreases $S_{l}\left(C E A, n_{h, i n}, n_{l, i n}\right)$,

(ii) an increase in $n_{l, \text { in }}$ strictly increases $S_{h}\left(C E A, n_{h, i n}, n_{l, i n}\right)$,

(iii) an increase in $n_{h, \text { in }}$ weakly increases $S_{l}\left(C E L, n_{h, i n}, n_{l, i n}\right)$,

(iv) an increase in $n_{h, \text { in }}$ strictly decreases $S_{h}\left(C E L, n_{h, i n}, n_{l, i n}\right)$,

(v) an increase in $n_{h, \text { in }}$ weakly increases $S_{l}\left(C E A, n_{h, i n}, n_{l, i n}\right)$,

(vi) an increase in $n_{h, \text { in }}$ strictly decreases $S_{h}\left(C E A, n_{h, i n}, n_{l, i n}\right)$,

(vii) an increase in $n_{l, \text { in }}$ weakly decreases $S_{l}\left(C E L, n_{h, i n}, n_{l, i n}\right)$, and

(viii) an increase in $n_{l, \text { in }}$ strictly increases $S_{h}\left(C E L, n_{h, i n}, n_{l, i n}\right)$.

Proof. See Appendix B.

The comparative statics stated in Lemma 5 have simple intuitions. In a nutshell, they show the changes in per-capita transfer with respect to changes in the number of type $h$ and type $l$ agents. We see that if the number of agents of types who are making transfers increases, per-capita transfers they make decrease and per-capita transfers other types receive increase. On the other hand, if the number of types who are receiving transfers increases, per-capita transfers they receive decrease and percapita transfers other types make increase. The following lemma states that under $P$, a type $l$ agent prefers to play in if and only if a type $h$ agent prefers to play $i n$.

Lemma 6 Given a bankruptcy problem $(C, E) \in \widetilde{\mathcal{B}}, P_{h} \geq V_{h}$ if and only if $P_{l} \geq V_{l}$.

Proof. See Appendix B.

Tie-Breaking Assumption Every agent plays in when he is indifferent between in and out.

This tie-breaking assumption is employed in the rest of the paper. The following lemma states that each agent's decision in equilibrium is determined by his type only. 
Lemma 7 (Symmetry) If agents $i$ and $j$ are of the same type $t \in\{l, h\}$, their strategies are the same in equilibrium.

\section{Proof. See Appendix B.}

Lemma 7 has three important implications. First of all, it shows that if there exists an equilibrium it will be symmetric, i.e., same types play the same strategy in equilibrium. The tie-breaking assumption is important for the validity of Lemma 7. If agents of the same type play strategies that are different from each other when these agents are indifferent, our statement is not valid anymore. However, breaking the ties in favor of playing in is not crucial for the proofs. Assuming that every agent plays out when he is indifferent would work equally well.

Second, this symmetry result enables us to employ a more compact notation for equilibrium actions in subgames $\Gamma^{F}$ (in game $\Gamma$ ): $\left(a_{h, F}, a_{l, F}\right)$ means that all type $h$ agents play $a_{h, F}$ and all type $l$ agents play $a_{l, F}$ in the subgame $\Gamma^{F}$.

Third, this result also enables us to use a simpler notation when writing agents' expected payoffs. Since we know that agents of the same type act identically, we can write the expected payoff of a representative type $t$ agent who plays $i n$ under rule $F$ as $V_{t}^{e}\left(F, s_{-t}\right)$ instead of writing individual expected payoff as $V_{i, i n}^{e}\left(F, n_{h, i n}, n_{l, i n}\right) .{ }^{10}$ We will employ this notation in the remaining part of the model.

The following corollary relates the symmetry result to the equilibrium values of $V_{m}(F)$. Since we show that agents of the same type have the same strategies in equilibrium, this reduces the number of possible values of equilibrium investment.

Corollary 3 In equilibrium, $V_{m}(F)$ can take only four values: $0, n_{h} w_{h}, n_{l} w_{l}$ and $n_{h} w_{h}+n_{l} w_{l}$.

Proof. Since there are two types of agents, by Lemma 7, there are four possible combinations of strategy profiles under the symmetry result proven above:

(i) Both types play out. Thus, $V_{m}(F)=0$,

(ii) Type $h$ agents play in, type $l$ agents play out. Thus, $V_{m}(F)=n_{h} w_{h}$,

(iii) Type $h$ agents play out, type $l$ agents play $i n$. Thus, $V_{m}(F)=n_{l} w_{l}$,

(iv) Both types play in. Thus, $V_{m}(F)=n_{h} w_{h}+n_{l} w_{l}$.

The following lemma shows that certain strategy profiles cannot exist in any equilibrium under $C E A$ and $C E L$.

Lemma 8 The following statements about strategy profiles are valid.

(i) In the subgame $\Gamma^{C E A}$, the strategy profile (for all $i \in N_{h}, \psi_{i}=$ in and for all $j \in N_{l}, \psi_{j}=$ out) cannot be an equilibrium,

(ii) In the subgame $\Gamma^{C E L}$, the strategy profile (for all $i \in N_{h}, \psi_{i}=$ out and for all $j \in N_{l}, \psi_{j}=$ in) cannot be an equilibrium.

\footnotetext{
${ }^{10}$ The equal treatment of equals property asserts that the agents with equal claims should receive the same payoff. This property is satisfied by all rules we consider here, which enables us to use this compact notation.
} 
Proof. See Appendix B.

The result in this lemma has a simple intuition: if, in equilibrium, the parameter values are such that even the type of agents who are making transfers find playing in optimal, the type of agents who are receiving transfers also find it optimal to play in.

\subsection{Characterization of All Nash Equilibria in $\Gamma^{P}, \Gamma^{C E A}$, and $\Gamma^{C E L}$}

In this subsection, we describe agents' investment behavior and characterize all Nash equilibria in subgames $\Gamma^{P}, \Gamma^{C E A}$, and $\Gamma^{C E L}$. Recall that Lemma 7 enables us to use type best responses instead of agent best responses. Hence, in this section, we use type $t$ 's best response to a strategy played by the other type. Denote the best response of type $t$ agents to action $a_{-t}$ played by the other type of agents in the subgame $\Gamma^{F}$ by $B R_{t}\left(F, a_{-t}\right){ }^{11}$

\section{Under the Proportional Rule:}

In the subgame $\Gamma^{P}$, the following payoff matrix can be used to show representative type $h$ and type $l$ agent's expected payoffs. The first (second) item in each cell represents each type $h$ (type $l$ ) agent's expected payoff. Matrices are drawn for representative agents of type $h$ and type $l$.

\begin{tabular}{|c|c|c|}
\hline$h \backslash l$ & in & out \\
\hline in & $P_{h}^{e}, P_{l}^{e}$ & $P_{h}^{e}, V_{l, \text { out }}$ \\
\hline out & $V_{h, \text { out }}, P_{l}^{e}$ & $V_{h, \text { out }}, V_{l, \text { out }}$ \\
\hline
\end{tabular}

Table 2: Payoff Matrix under the Proportional Rule

Recall that by Lemma 3, the expected payoff of each agent is independent of other agents' strategies under $P$. This implies that all equilibria are dominant strategy equilibria. Also, remember that by Lemma $6, P_{l}^{e}<V_{l, \text { out }}$ if and only if $P_{h}^{e}<V_{h, \text { out }}$. Therefore, if $P_{h}^{e} \geq V_{h \text {,out }}$, then $B R_{h}(P$, in $)=B R_{h}(P$, out $)=i n$, and similarly if $P_{l}^{e} \geq V_{l, \text { out }}$, then $B R_{l}(P$, in $)=B R_{l}(P$, out $)=i n$. If $P_{h}^{e}<V_{h, \text { out }}$, then $B R_{h}(P$, in $)=$ $B R_{h}(P$, out $)=$ out, and similarly if $P_{l}^{e}<V_{l, \text { out }}$, then $B R_{l}(P$, in $)=B R_{l}(P$, out $)=$ out. Also recall that $\left(a_{h, F}, a_{l, F}\right)$ means that all type $h$ agents play $a_{h, F}$ and all type $l$ agents play $a_{l, F}$ in the subgame $\Gamma^{F}$. We now describe agents' equilibrium strategies in the subgame $\Gamma^{P}$.

Equilibria in the subgame $\Gamma^{P}$ :

\footnotetext{
${ }^{11}$ Also note that each agent has one information set in each subgame and two actions. Therefore, the terms strategy and action refer to same objects in subgames $\Gamma^{P}, \Gamma^{C E A}$, and $\Gamma^{C E L}$. Hence, we prefer to stick to action notation instead of introducing strategy notation for subgames, although we use the terms strategy and action interchangeably.
} 
Case 1 If for all $t \in\{l, h\}, P_{t}^{e}<V_{t, o u t}$, then the unique equilibrium strategy profile is $\left(a_{h, P}, a_{l, P}\right)=($ out, out $)$.

Case 2 If for all $t \in\{l, h\}, P_{t}^{e} \geq V_{t, \text { out }}$, then the unique equilibrium strategy profile is $\left(a_{h, P}, a_{l, P}\right)=(i n, i n)$.

Note that neither (in, out) nor (out,in) equilibria are possible. This is due to proportionality, which implies that $P_{h}^{e} \geq V_{h, \text { out }}$ if and only if $P_{l}^{e} \geq V_{l, \text { out }}$.

\section{Under the Constrained Equal Awards Rule:}

In the subgame $\Gamma^{C E A}$ :

\begin{tabular}{|c|c|c|}
\hline$h \backslash l$ & in & out \\
\hline in & $\begin{array}{c}P_{h}^{e}+\left(1-\pi_{s}\right) S_{h}(C E A, \text { in }), \\
P_{l}^{e}+\left(1-\pi_{s}\right) S_{l}(C E A, \text { in })\end{array}$ & $P_{h}^{e}, V_{l, \text { out }}$ \\
\hline out & $V_{h, \text { out }}, P_{l}^{e}$ & $V_{h, \text { out }}, V_{l, \text { out }}$ \\
\hline
\end{tabular}

Table 3: Payoff Matrix under the Constrained Equal Awards Rule

By the definition of the constrained equal awards rule and Lemma 1, $S_{h}(C E A$, in $)<$ 0 and $S_{l}(C E A, i n)>0$. If the outside asset pays more than the best possible expected payoff that type $l$ agents can get, the analysis is trivial since then type $l$ agents would never play in. Hence, we assume that $V_{l}^{e}(C E A, i n)=P_{l}^{e}+\left(1-\pi_{s}\right) S_{l}(C E A, i n)>$ $V_{l, \text { out }}$. This assumption implies that $B R_{l}(P, i n)=i n$. The relationship between $P_{l}^{e}$ and $V_{l, \text { out }}$ determines type $l$ agents' best response to type $h$ agents playing out. If $P_{l}^{e} \geq V_{l}$, then $B R_{l}(P$, out $)=$ in; if $P_{l}<V_{l, \text { out }}$, then $B R_{l}(P$, out $)=$ out. On the other hand, type $h$ 's best response against in depends on the relationship between $V_{h}^{e}(C E A, i n)=P_{h}^{e}+\left(1-\pi_{s}\right) S_{h}(C E A$, in $)$ and $V_{h, o u t}$. If

$$
V_{h}^{e}(C E A, \text { in })=P_{h}^{e}+\left(1-\pi_{s}\right) S_{h}(C E A, \text { in }) \geq V_{h, \text { out }},
$$

then $B R_{h}(P, i n)=i n$; if

$$
V_{h}^{e}(C E A, \text { in })=P_{h}^{e}+\left(1-\pi_{s}\right) S_{h}(C E A, \text { in })<V_{h, \text { out }},
$$

then $B R_{h}(P$, in $)=$ out. Therefore, these inequalities characterize agents' equilibrium strategies in the subgame $\Gamma^{C E A}$.

Equilibria in the subgame $\Gamma^{C E A}$ :

Case 1 If for all $t \in\{l, h\}, P_{t}^{e}<V_{t, \text { out }}$, then the unique equilibrium strategy profile is $\left(a_{h, C E A}, a_{l, C E A}\right)=($ out, out $)$.

Case 2 If for all $t \in\{l, h\}, P_{t}^{e} \geq V_{t, \text { out }}$ and $V_{h}^{e}(C E A$, in $)=P_{h}^{e}+\left(1-\pi_{s}\right) S_{h}(C E A$, in $)<$ $V_{h, o u t}$, then the unique equilibrium strategy profile is $\left(a_{h, C E A}, a_{l, C E A}\right)=($ out, in $)$. 
Case 3 If for all $t \in\{l, h\}, P_{t}^{e} \geq V_{t, \text { out }}$ and $V_{h}^{e}(C E A$, in $)=P_{h}^{e}+\left(1-\pi_{s}\right) S_{h}(C E A$, in $) \geq$ $V_{h, o u t}$, then the unique equilibrium strategy profile is $\left(a_{h, C E A}, a_{l, C E A}\right)=($ in, in $)$.

\section{Under the Constrained Equal Losses Rule:}

In the subgame $\Gamma^{C E L}$ :

\begin{tabular}{|c|c|c|}
\hline$h \backslash l$ & in & out \\
\hline in & $\begin{array}{c}P_{h}^{e}+\left(1-\pi_{s}\right) S_{h}(C E L, \text { in }), \\
P_{l}^{e}+\left(1-\pi_{s}\right) S_{l}(C E L, \text { in })\end{array}$ & $P_{h}^{e}, V_{l, \text { out }}$ \\
\hline out & $V_{h, \text { out }}, P_{l}^{e}$ & $V_{h, \text { out }}, V_{l, \text { out }}$ \\
\hline
\end{tabular}

Table 4: Payoff Matrix under the Constrained Equal Losses Rule

By the definition of the constrained equal losses rule and Lemma $1, S_{h}(C E L$, in $)>$ 0 and $S_{l}(C E L, i n)<0$. If the outside asset pays more than the best possible expected payoff that type $h$ agents can get, the analysis is trivial since then type $h$ agents would never play in. Hence, we assume that $V_{h}^{e}(C E L, i n)=P_{h}^{e}+\left(1-\pi_{s}\right) S_{h}(C E L, i n)>$ $V_{h, o u t}$. This assumption implies that $B R_{h}(P, i n)=i n$. The relationship between $P_{h}^{e}$ and $V_{h \text {,out }}$ determines type $h$ agents' best response to type $l$ agents playing out. If $P_{h}^{e} \geq V_{h}$, then $B R_{h}(P$, out $)=$ in; if $P_{h}<V_{h, \text { out }}$, then $B R_{h}(P$, out $)=$ out. On the other hand, type l's best response to type $h$ agents playing in depends on the relationship between $V_{l}^{e}(C E L, i n)=P_{l}^{e}+\left(1-\pi_{s}\right) S_{l}(C E L$, in $)$ and $V_{l, o u t}$. If

$$
V_{l}^{e}(C E L, \text { in })=P_{l}^{e}+\left(1-\pi_{s}\right) S_{l}(C E L, \text { in }) \geq V_{l, o u t},
$$

then $B R_{l}(P, i n)=i n$; if

$$
V_{l}^{e}(C E L, \text { in })=P_{l}^{e}+\left(1-\pi_{s}\right) S_{l}(C E L, \text { in })<V_{l, \text { out }},
$$

then $B R_{l}(P$, in $)=$ out. Therefore, these inequalities characterize agents' equilibrium actions under the constrained equal losses rule.

Equilibria in the subgame $\Gamma^{C E L}$ :

Case 1 If for all $t \in\{l, h\}, P_{t}^{e}<V_{t, o u t} \Gamma^{C E L}$, then the unique equilibrium strategy profile is $\left(a_{h, C E L}, a_{l, C E L}\right)=($ out, out $)$.

Case 2 If for all $t \in\{l, h\}, P_{t}^{e} \geq V_{t, \text { out }}$ and $V_{l}^{e}(C E L$, in $)=P_{l}^{e}+\left(1-\pi_{s}\right) S_{l}(C E L$, in $)<$ $V_{l, o u t}$, then the unique equilibrium strategy profile is $\left(a_{h, C E L}, a_{l, C E L}\right)=($ in, out $)$.

Case 3 If for all $t \in\{l, h\}, P_{t}^{e} \geq V_{t, \text { out }}$ and $V_{l}^{e}(C E L, i n)=P_{l}^{e}+\left(1-\pi_{s}\right) S_{l}(C E L$, in $) \geq$ $V_{l, \text { out }}$, then the unique equilibrium strategy profile is $\left(a_{h, C E L}, a_{l, C E L}\right)=($ in, in $)$.

Note that in a Nash equilibrium of the subgame $\Gamma^{C E A}$, if type $h$ agents choose to play in, type $l$ agents also choose to play $i n$. Similarly, in a Nash equilibrium of the subgame $\Gamma^{C E L}$, if type $l$ agents choose to play $i n$, type $h$ agents also choose to play $i n$. Also note that, if the equilibrium of the subgame $\Gamma^{P}$ is the strategy profile $\left(a_{h, P}, a_{l, P}\right)=($ out, out $)$, then the equilibrium strategy profiles of the subgames $\Gamma^{C E A}$ and $\Gamma^{C E L}$ are also $\left(a_{h, C E A}, a_{l, C E A}\right)=\left(a_{h, C E L}, a_{l, C E L}\right)=($ out, out $)$. 


\subsection{Characterization of All Subgame Perfect Nash Equilibria in $\Gamma$}

Having finished analyzing agents' behavior in all three subgames, we analyze the company's behavior and characterize all subgame perfect Nash equilibria in $\Gamma$ in this subsection. As we mentioned in Section 3, the company's payoff function is $V_{m}(F)=\sum_{t \in\{l, h\}} n_{t, i n} w_{t}$ where $n_{t, i n}$ is the number of type $t$ agents played $i n$. Therefore, the company's decision depends on the equilibrium strategies of agents in each subgame and the resulting level of investment. In the previous section, we analyzed the equilibrium strategies of agents in all three subgames. Below, we list different combinations of inequalities and the subgame perfect Nash equilibrium strategy profiles along with the equilibrium investment in the company. In the strategy profile $\left(s_{m}, s_{h}, s_{l}\right)$, the first entry refers to the company's strategy (i.e., $\left.s_{m} \in \psi_{m}\right)$, second to type $h$ agents' (i.e., $s_{h} \in \psi_{h}$ ), and third to type $l$ agents' (i.e., $s_{l} \in \psi_{l}$ ). Moreover, the first entry in a representative type $t$ agent's strategy profile refer to his equilibrium action in $\Gamma^{P}$, the second to his equilibrium action in $\Gamma^{C E A}$, and the third to his equilibrium action in $\Gamma^{C E L}$.

C1. If for all $t \in\{l, h\}$

$$
\begin{aligned}
P_{t}^{e} & \geq V_{t, \text { out }}, \\
P_{h}^{e}+\left(1-\pi_{s}\right) S_{h}(C E A, \text { in }) & <V_{h, \text { out }}, \text { and } \\
P_{l}^{e}+\left(1-\pi_{s}\right) S_{l}(C E L, \text { in }) & <V_{l, \text { out }},
\end{aligned}
$$

then given the agents' equilibrium actions in subgames $\Gamma^{P}, \Gamma^{C E A}$, and $\Gamma^{C E L}$ presented in the previous subsection, the company prefers the proportional rule and the equilibrium investment in the company is $V_{m}(F)=\sum_{t \in\{l, h\}} n_{t} w_{t}$. As we showed in the previous subsection, under these parameter restrictions, neither the constrained equal awards rule nor the constrained equal losses rule can attract all types to invest in the company, whereas the proportional rule can. Hence, the unique subgame perfect Nash equilibrium strategy profile is

$$
\left(s_{m}, s_{h}, s_{l}\right)=(P,(\text { in } ; \text { out } ; \text { in }),(\text { in } ; \text { in } ; \text { out })) .
$$

C2. If for all $t \in\{l, h\}$,

$$
\begin{aligned}
P_{t}^{e} & \geq V_{t, \text { out }}, \\
P_{h}^{e}+\left(1-\pi_{s}\right) S_{h}(C E A, \text { in }) & \geq V_{h, \text { out }}, \text { and } \\
P_{l}^{e}+\left(1-\pi_{s}\right) S_{l}(C E L, \text { in }) & <V_{l, \text { out }},
\end{aligned}
$$

then given the agents' equilibrium actions in subgames $\Gamma^{P}, \Gamma^{C E A}$, and $\Gamma^{C E L}$ presented in the previous subsection, the company prefers the proportional rule or the constrained equal awards rule to the constrained equal losses rule and the equilibrium investment in the company is $V_{m}(F)=\sum_{t \in\{l, h\}} n_{t} w_{t}$. As we 
showed in the previous subsection, under these parameter restrictions, both the constrained equal awards and the proportional rules can attract all types to invest in the company whereas the constrained equal losses rule can only attract $h$ types. Hence, the subgame perfect Nash equilibrium strategy profiles are

$$
\begin{aligned}
& \left(s_{m}, s_{h}, s_{l}\right)=(P,(\text { in } ; \text { in } ; \text { in }),(\text { in } ; \text { in } ; \text { out })) \text { and } \\
& \left(s_{m}, s_{h}, s_{l}\right)=(C E A,(\text { in } ; \text { in } ; \text { in }),(\text { in } ; \text { in } ; \text { out }))
\end{aligned}
$$

C3. If for all $t \in\{l, h\}$,

$$
\begin{aligned}
P_{t}^{e} & \geq V_{t, \text { out }}, \\
P_{h}^{e}+\left(1-\pi_{s}\right) S_{h}(C E A, \text { in }) & <V_{h, \text { out }}, \text { and } \\
P_{l}^{e}+\left(1-\pi_{s}\right) S_{l}(C E L, \text { in }) & \geq V_{l, \text { out }},
\end{aligned}
$$

then given the agents' equilibrium actions in subgames $\Gamma^{P}, \Gamma^{C E A}$, and $\Gamma^{C E L}$ presented in the previous subsection, the company prefers the proportional rule or the constrained equal losses rule to the constrained equal awards rule and the equilibrium investment in the company is $V_{m}(F)=\sum_{t \in\{l, h\}} n_{t} w_{t}$. As we showed in the previous subsection, under these parameter restrictions, the constrained equal losses and the proportional rules can attract all types to invest in the company whereas the constrained equal awards rule can only attract $l$ types. Hence, the subgame perfect Nash equilibrium strategy profiles are

$$
\begin{aligned}
& \left(s_{m}, s_{h}, s_{l}\right)=(P,(\text { in } ; \text { out } ; \text { in }),(\text { in } ; \text { in } ; \text { in })) \text { and } \\
& \left(s_{m}, s_{h}, s_{l}\right)=(C E L,(\text { in } ; \text { out } ; \text { in }),(\text { in } ; \text { in } ; \text { in })) .
\end{aligned}
$$

C4. If for all $t \in\{l, h\}$,

$$
\begin{aligned}
P_{t}^{e} & \geq V_{t, \text { out }}, \\
P_{h}^{e}+\left(1-\pi_{s}\right) S_{h}(C E A, \text { in }) & \geq V_{h, \text { out }}, \text { and } \\
P_{l}^{e}+\left(1-\pi_{s}\right) S_{l}(C E L, \text { in }) & \geq V_{l, \text { out }},
\end{aligned}
$$

then given the agents' equilibrium actions in subgames $\Gamma^{P}, \Gamma^{C E A}$, and $\Gamma^{C E L}$ presented in the previous subsection, the company is indifferent between all three rules, and the equilibrium investment in the company is $V_{m}(F)=\sum_{t \in\{l, h\}} n_{t} w_{t}$. As we showed in the previous subsection, under these parameter restrictions, all rules are equally able to attract all types to invest in the company. Hence, the subgame perfect Nash equilibrium strategy profiles are 


$$
\begin{aligned}
& \left(s_{m}, s_{h}, s_{l}\right)=(P,(i n ; i n ; i n),(i n ; i n ; i n)), \\
& \left(s_{m}, s_{h}, s_{l}\right)=(C E A,(i n ; i n ; i n),(i n ; i n ; i n)), \text { and } \\
& \left(s_{m}, s_{h}, s_{l}\right)=(C E L,(i n ; i n ; i n),(i n ; i n ; i n)) .
\end{aligned}
$$

C5. If for all $t \in\{l, h\}$,

$$
P_{t}^{e}<V_{t, \text { out }}
$$

then given the agents' equilibrium actions in subgames $\Gamma^{P}, \Gamma^{C E A}$, and $\Gamma^{C E L}$ presented in the previous subsection, the company is indifferent between all three rules, and the equilibrium investment in the company is $V_{m}(F)=0$. Since $P_{t}^{e}<V_{t, o u t}$ is a necessary condition for equilibrium decisions to be $(i n, i n)$, none of the rules can attract neither of the two types to invest in the company. Hence, the subgame perfect Nash equilibrium strategy profiles are

$$
\begin{aligned}
& \left(s_{m}, s_{h}, s_{l}\right)=(P,(\text { out } ; \text { out } ; \text { out }),(\text { out } ; \text { out } ; \text { out })) \\
& \left(s_{m}, s_{h}, s_{l}\right)=(\text { CEA },(\text { out } ; \text { out } \text { out }),(\text { out } ; \text { out } ; \text { out })), \text { and } \\
& \left(s_{m}, s_{h}, s_{l}\right)=(\text { CEL },(\text { out } ; \text { out } ; \text { out }),(\text { out } ; \text { out } ; \text { out })) .
\end{aligned}
$$

Note that in C4 and C5 above, the company's decision does not really matter. Basically, in these cases, anything goes. As we have shown above, besides $P_{t}^{e} \geq V_{t, \text { out }}$,

$$
\begin{aligned}
P_{h}^{e}+\left(1-\pi_{s}\right) S_{h}(C E A, \text { in }) & \geq V_{h, \text { out }} \text { and } \\
P_{l}^{e}+\left(1-\pi_{s}\right) S_{l}(C E L, \text { in }) & \geq V_{l, \text { out }}
\end{aligned}
$$

should be satisfied in $\mathrm{C} 4$. The interpretation of this is that neither under $C E A$ nor under $C E L$, per-capita transfers from disadvantaged type of agents to advantaged type of agents are significantly high. This intuitively means that the difference between $w_{l}$ and $w_{h}$ is not significantly large in $\mathrm{C} 4$.

C5 shows another situation in which the decision will not make a difference. No matter which rule the company chooses, the investment in the company will be 0 . However, this has nothing to do with the income distribution in the society. We already showed in Lemma 6 that $P_{t}^{e} \geq V_{t, \text { out }}$ does not contain any income distribution parameters (e.g., $n_{l}, n_{h}, w_{l}$ and $w_{h}$ ). Hence, the validity of this condition depends only on the risk-return characteristics of investment alternatives. Intuitively, if the payoff from the risk-free asset is sufficiently high, or the failure probability of the risky investment project is sufficiently high (or more generally the expected return from the risky investment is sufficiently low) then $P_{t}^{e}<V_{t, \text { out }}$ will hold, in which case the company's decision cannot change the equilibrium investment in the company. We analyze the effect of changes in the parameters on the equilibrium and the corresponding investment in the company in more detail in Section 5. 


\subsection{Equilibrium and Results}

In Subsections 4.1 and 4.2, we analyzed agents' and the company's decisions and characterized all subgame perfect Nash equilibria. In this subsection, we present some results which are implied by that equilibrium analysis. The following proposition states that there always exists a pure strategy subgame perfect Nash equilibrium of game $\Gamma$.

Proposition 1 A pure strategy subgame perfect Nash equilibrium of $\Gamma$ exists.

Proof. Follows from the analyses in Subsections 4.1 and 4.2.

Below we present our main result. It shows that the proportional rule has a very strong position in our noncooperative setting.

Theorem 1 For any bankruptcy problem $(C, E) \in \widetilde{\mathcal{B}}$, there always exists a subgame perfect Nash equilibrium of $\Gamma$, which involves the proportional rule.

Proof. Notice that in all five cases analyzed in Subsection 4.2, the subgame perfect Nash equilibria involves the proportional rule. Since, we characterized all equilibria in Subsection 4.2, the result immediately follows.

Notice that in C3, the subgame perfect Nash Equilibria do not involve the constrained equal awards rule, in C2, the subgame perfect Nash equilibria do not involve the constrained equal losses rule and in C1, the subgame perfect Nash Equilibria do not involve neither of these two. Hence, the statement in the main theorem is valid only for the proportional rule.

The following corollary shows that our main result is robust with respect to the income distribution.

Corollary 4 The statement in the main theorem is valid independent of the income distribution.

Proof. Take any income distribution characterized by the parameters, $n_{h}, n_{l}, w_{h}$ and $w_{l}$. The condition that determines equilibrium under $P$ is: for all $t \in\{l, h\}, P_{t}^{e} \geq$ $V_{t, \text { out }}$, which is independent of the income distribution parameters as shown in Lemma 6. Since $P_{h}^{e}+\left(1-\pi_{s}\right) S_{h}(C E A$, in $) \geq V_{h, \text { out }}$ and $P_{l}^{e}+\left(1-\pi_{s}\right) S_{l}(C E L$, in $) \geq V_{l, \text { out }}$ depend on income distribution parameters, the statement of the corollary cannot be valid for $C E A$ and $C E L$.

Theorem 1 and Corollary 4 provide a justification from a noncooperative point of view for the fact that the proportional rule is frequently employed in allocating a bankrupt company's assets to shareholders.

By Corollary 4, even if there is an uncertainty about the income distribution (i.e., the company does not know the income distribution for sure) statements in the Theorem 1 are still valid. In fact, for probability distributions that assign non-zero probability to all possible income distributions, the proportional rule would be the unique optimal strategy for an expected-payoff maximizing company. Also, note that by Theorem 1 and Corollary 4, the average investment in the company under the proportional rule is largest among the three rules. 


\subsection{Extension to a Larger Set of Rules}

In this subsection, we show that our results remain valid if we enlarge the set of rules we use in our model. Our first candidate is the Talmud rule since it is one of the prominent bankruptcy rules, along with three rules analyzed above and satisfies a large set of "desirable" properties (see Herrero \& Villar, 2001 and Thomson, 2006). Aumann \& Maschler (1985), in a seminal article, propose the Talmud rule as a consistent extension of the contested garment rule.

Definition 5 (Talmud Rule) For all $(C, E) \in \widetilde{\mathcal{B}}$, and all $j \in N$,

$$
T A L_{j}(C, E) \equiv\left\{\begin{array}{c}
\min \left\{\frac{c_{j}}{2}, \lambda_{t}\right\} \text { if } E \leq \sum_{i \in N} \frac{c_{i}}{2} \\
c_{j}-\min \left\{\frac{c_{j}}{2}, \lambda_{t}\right\} \text { if } E \geq \sum_{i \in N} \frac{c_{i}}{2}
\end{array}\right.
$$

where $\lambda_{t}$ solves $\sum_{i \in N} T A L_{i}(C, E)=E$.

Note that the Talmud rule coincides with the constrained equal awards rule if $E \leq \sum_{i \in N} \frac{c_{i}}{2}$ and with the constrained equal losses rule if $E \geq \sum_{i \in N} \frac{c_{i}}{2}$ (see MorenoTernero \& Villar, 2006). When $E=\sum_{i \in N} \frac{c_{i}}{2}$, the proportional rule, the constrained equal awards rule and the constrained equal losses rule lead to the same awards vector. In our model, the relative value of $E$ with respect to $\sum_{i \in N} \frac{c_{i}}{2}$ is completely determined by values of $r$ and $r_{f}$. For instance, $E<\sum_{i \in N} \frac{c_{i}}{2}$ corresponds to $1<r-2 r_{f}$, $E=\sum_{i \in N} \frac{c_{i}}{2}$ to $r=1$ and $r_{f}=0$, and $E>\sum_{i \in N} \frac{c_{i}}{2}$ to $r-2 r_{f}<1$. Since we do not allow $r_{f}<0$ or $r>1, E<\sum_{i \in N} \frac{c_{i}}{2}$ is not possible. Therefore, the Talmud rule coincides with the constrained equal losses rule in our model, which trivially implies that including the Talmud rule does not change our results.

Moreno-Ternero \& Villar (2006) generalize the Talmud rule by introducing the TAL-family of rules, which contains the Talmud, constrained equal awards and the constrained equal losses rules as special cases.

Definition 6 (TAL-family) The TAL-family consists of all rules of the following form: For some $\theta \in[0,1]$, for all $(E, C) \in \widetilde{\mathcal{B}}$ and for all $i \in N$,

$$
F_{i}^{\theta}(C, E)=\left\{\begin{array}{c}
\min \left\{\theta c_{i}, \lambda\right\} \text { if } E \leq \sum_{i \in N} \theta c_{i} \\
\max \left\{\theta c_{i}, c_{i}-\mu\right\} \text { if } E \geq \sum_{i \in N} \theta c_{i}
\end{array}\right.
$$

where $\mu$ and $\lambda$ solve $\sum_{i \in N} F_{i}^{\theta}(C, E)=E$.

Note that $F=C E L$ for $\theta=0, F=C E A$ for $\theta=1$ and $F=T$ for $\theta=1 / 2$. On the other hand, $P$ is not a member of the TAL-family. Moreover, for all $\theta \in\left[0, \frac{1}{2}\right]$, $F^{\theta}$ coincides with the constrained equal losses rule in our model and for all $\theta \in\left[\frac{1}{2}, 1\right]$, $F^{\theta}$ coincides with the constrained equal awards rule if $E \leq \sum_{i \in N} \theta c_{i}$ and with the constrained equal losses rule if $E \geq \sum_{i \in N} \theta c_{i}$ (see Moreno-Ternero \& Villar, 2006).

Now, we change the game by including all members of the TAL-family along with the proportional rule in the strategy space of the company. Therefore, $\Gamma$ has infinitely many subgames which are $\Gamma^{P}$ and, for all $\theta \in[0,1], \Gamma^{\theta}$. 
Theorem 2 For any bankruptcy problem $(E, C) \in \widetilde{\mathcal{B}}$, there always exists a subgame perfect Nash equilibrium of $\Gamma$ with the proportional rule and the member of the TALfamily parametrized by $\theta^{*}=\frac{1+r_{f}}{1+r}$.

Proof. Given $r$ and $r_{f}, \frac{E}{\sum_{i \in N} c_{i}}=\frac{1+r_{f}}{1+r} \in\left[\frac{1}{2}, 1\right)$. There exists a $\theta^{*} \in\left[\frac{1}{2}, 1\right]$ such that $\theta^{*}=\frac{1+r_{f}}{1+r}$. The awards vector under rule $F^{\theta^{*}}$ coincides with the awards vector under the proportional rule. Any other member of the TAL-family coincides either with the constrained equal awards rule or the constrained equal losses rule given $r$ and $r_{f}$. Therefore, the result follows from Theorem 1.

\section{Comparative Static Analysis}

In this section, we conduct comparative static analyses on the risk-return parameters and income distribution parameters. We show how the equilibrium investment in the company responds to changes in these parameters. First of all, we present the closed form versions of the inequalities that determine the equilibrium under each rule. This will help us in conducting comparative static analyses.

Recall that we have only (in, in) and (out,out) as (representative) subgame perfect Nash equilibrium strategy profiles. Therefore, in the following we provide the closed form expressions of the inequalities that lead to these subgame perfect equilibria.

The Proportional Rule:

For $(i n, i n)$ equilibrium,

$$
P_{t}^{e} \geq V_{t, \text { out }}
$$

is required. It can be written explicitly as

$$
\begin{aligned}
\pi_{s}(1+r) w_{t}+\left(1-\pi_{s}\right)\left(1+r_{f}\right) w_{t} & \geq(1+\bar{r}) w_{t} \\
\pi_{s} r+r_{f}-\pi_{s} r_{f} & \geq \bar{r} \\
\pi_{s}\left(r-r_{f}\right) & \geq \bar{r}-r_{f} \\
\pi_{s} & \geq \frac{\bar{r}-r_{f}}{r-r_{f}} .
\end{aligned}
$$

Obviously, $\pi_{s}<\frac{\bar{r}-r_{f}}{r-r_{f}}$ leads to (out, out) equilibrium. Moreover, recall that $\pi_{s}<\frac{\bar{r}-r_{f}}{r-r_{f}}$ also leads to (out, out) equilibrium under $C E A$ and $C E L$.

The Constrained Equal Awards Rule:

For $(i n, i n)$ equilibrium

$$
\begin{gathered}
P_{t}^{e} \geq V_{t, \text { out }} \text { and } \\
P_{h}^{e}+\left(1-\pi_{s}\right) S_{h}(C E A, \text { in }) \geq V_{h, \text { out }}
\end{gathered}
$$


is required. We consider two cases to express the second inequality explicitly under $C E A$ :

(a-1) If $c_{h}>c_{l}>\lambda_{\text {cea }}$, then $P_{h}^{e}+\left(1-\pi_{s}\right) S_{h}(C E A$, in $) \geq V_{h, \text { out }}$ can be written as,

$\pi_{s}(1+r) w_{h}+\left(1-\pi_{s}\right)\left(1+r_{f}\right) w_{h}+\left(1-\pi_{s}\right) \frac{n_{l}}{n_{h}+n_{h}}\left[\left(1+r_{f}\right)\left(w_{l}-w_{h}\right)\right] \geq(1+\bar{r}) w_{h}$,

which is equal to

$$
\frac{n_{l}}{n_{l}+n_{h}} \leq \frac{w_{h}}{w_{h}-w_{l}} \frac{\pi_{s} r+\left(1-\pi_{s}\right) r_{f}-\bar{r}}{\left(1-\pi_{s}\right)\left(1+r_{f}\right)}
$$

(a-2) If $c_{h}>\lambda_{\text {cea }} \geq c_{l}$, then $P_{h}^{e}+\left(1-\pi_{s}\right) S_{h}(C E A$, in $) \geq V_{h, \text { out }}$ can be written as,

$$
\pi_{s}(1+r) w_{h}+\left(1-\pi_{s}\right)\left(1+r_{f}\right) w_{h}+\left(1-\pi_{s}\right) \frac{n_{l}}{n_{h}}\left(r_{f}-r\right) w_{l} \geq(1+\bar{r}) w_{h},
$$

which is equal to

$$
\frac{n_{l}}{n_{h}} \leq \frac{w_{h}}{w_{l}} \frac{\pi_{s} r+\left(1-\pi_{s}\right) r_{f}-\bar{r}}{\left(1-\pi_{s}\right)\left(r-r_{f}\right)}
$$

The Constrained Equal Losses Rule:

For $(i n, i n)$ equilibrium

$$
\begin{gathered}
P_{t}^{e} \geq V_{t, \text { out }} \text { and } \\
P_{l}^{e}+\left(1-\pi_{s}\right) S_{l}(C E L, \text { in }) \geq V_{l, \text { out }}
\end{gathered}
$$

is required. We consider two cases to express the second inequality explicitly under $C E L$ :

(b-1) If $c_{h}>c_{l}>\lambda_{c e l}$, then $P_{l}^{e}+\left(1-\pi_{s}\right) S_{l}(C E L$, in $) \geq V_{l, o u t}$ can be written as,

$$
\pi_{s}(1+r) w_{l}+\left(1-\pi_{s}\right)\left(1+r_{f}\right) w_{l}+\left(1-\pi_{s}\right) \frac{n_{h}}{n_{h}+n_{l}}\left[\left(r-r_{f}\right)\left(w_{l}-w_{h}\right)\right] \geq(1+\bar{r}) w_{l}
$$

which is equal to

$$
\frac{n_{h}}{n_{h}+n_{l}} \leq \frac{w_{l}}{w_{h}-w_{l}} \frac{\pi_{s} r+\left(1-\pi_{s}\right) r_{f}-\bar{r}}{\left(1-\pi_{s}\right)\left(r-r_{f}\right)}
$$

(b-2) If $c_{h}>\lambda_{\text {cea }} \geq c_{l}$, then $P_{l}^{e}+\left(1-\pi_{s}\right) S_{l}(C E L$, in $) \geq V_{l, \text { out }}$ can be written as,

$$
\pi_{s}(1+r) w_{l} \geq(1+\bar{r}) w_{l},
$$

which is equal to

$$
\pi_{s} \geq \frac{1+\bar{r}}{1+r}
$$


Note that $\pi_{s} \geq \frac{\bar{r}-r_{f}}{r-r_{f}}$ is already implicitly embedded in (8), (9), (10) and (11) also implies it. Hence practically, we do not have two conditions determining the (in, in) equilibrium under $C E A$ and $C E L$. Below, we present a table consisting of parameters $r, r_{f}, \bar{r}, \pi_{s}, n_{l}, n_{h}, w_{l}$, and $w_{h}$ and the effects of changes in those on the inequalities that determine the subgame perfect equilibrium investment in the company. In particular, + in a cell means that the inequality in the corresponding column will still be valid after an increase in the respective parameter and - in a cell means that the inequality in the corresponding column might not be valid after an increase.

\begin{tabular}{||c|c|c|c|c|c||}
\hline \hline & $(7)$ & $(8)$ & $(9)$ & $(10)$ & $(11)$ \\
\hline$r$ & + & + & + & + & + \\
\hline$r_{f}$ & + & + & + & + & no change \\
\hline $\bar{r}$ & - & - & - & - & - \\
\hline$\pi_{s}$ & + & + & + & + & + \\
\hline$n_{l}$ & no change & - & - & + & no change \\
\hline$n_{h}$ & no change & + & + & - & no change \\
\hline$w_{l}$ & no change & + & - & + & no change \\
\hline$w_{h}$ & no change & - & + & - & no change \\
\hline \hline
\end{tabular}

Table 5: Comparative Static Analysis on the Set of SPNE

The following proposition shows the relationship between risk-return parameters and the equilibrium investment in the company.

Proposition $2 A$ change in $r, r_{f}, \bar{r}$ and $\pi$ can lead to only two types of movements in the equilibrium investment in the company:

(i) Either the equilibrium investment in the company decreases from $\sum_{t \in\{l, h\}} n_{t} w_{t}$ to 0 , or

(ii) the equilibrium investment in the company increases from 0 to $\sum_{t \in\{l, h\}} n_{t} w_{t}$.

Proof. In all subgame perfect Nash equilibria, there are only two possible levels of investment: 0 and $\sum_{t \in\{l, h\}} n_{t} w_{t}$. Hence, if a change in $r, r_{f}, \bar{r}$ and $\pi$ causes a change in the equilibrium investment it can be either a movement from $\sum_{t \in\{l, h\}} n_{t} w_{t}$ to 0 , or a movement from 0 to $\sum_{t \in\{l, h\}} n_{t} w_{t}$.

Proposition 3 An increase in $r, r_{f}$ or $\pi_{s}$ can never decrease the equilibrium investment in the company.

Proof. The proof is simple and hence omitted. It follows from the fact that agents' expected payoff from playing in is increasing in $r, r_{f}$ and $\pi_{s}$.

This result also shows that taxes on $r$ and/or $r_{f}$ can be used as a policy tool by the government to increase the shareholders in the company. By decreasing the taxes, the government can increase the number of shareholders as well as the investment in 
the company. On the other hand, the return from the risk-free outside asset $(\bar{r})$ enters all inequalities above from the right side with a positive sign. Therefore, an increase in $\bar{r}$ increases the attractiveness of the risk-free asset i.e. playing out. Accordingly, the equilibrium investment in the company weakly decreases as a result of an increase in $\bar{r}$. Similar to the policy implication about $r$ and $r_{f}$ above, taxes on $\bar{r}$ can also be used as a policy tool. The government can increase the investment in the company by increasing the taxes on the risk-free asset.

The following proposition shows the relationship between income distribution parameters and the equilibrium investment in the company.

Proposition 4 Decreases in $n_{l}, n_{h}, w_{l}$ and $w_{h}$ can never increase the equilibrium investment in the company.

Proof. The proof is simple and follows from the following facts:

(i) The proportional rule is always a part of the equilibrium.

(ii) The condition determining the equilibrium strategies under the proportional rule is independent of the income distribution parameters (i.e., $n_{l}, n_{h}, w_{l}$ and $w_{h}$ ) as shown in Lemma 6.

(iii) If $P_{t}^{e}<V_{t, \text { out }}$, the equilibrium investment in the company is 0 and does not change due to any change in $n_{l}, n_{h}, w_{l}$ and $w_{h}$.

(iv) If $P_{t}^{e} \geq V_{t, \text { out }}$, the equilibrium investment in the company under $P$ is $\sum_{t \in\{l, h\}} n_{t} w_{t}$. Since the change in $\sum_{t \in\{l, h\}} n_{t} w_{t}$ with respect to a change in $n_{l}, n_{h}, w_{l}$ or $w_{h}$, a decrease in any of these parameters decreases the equilibrium investment in the company.

Needless to say, increases in $n_{l}, n_{h}, w_{l}$ and $w_{h}$ can never decrease the equilibrium investment in the company.

The following corollary shows the relationship between the impact a change in parameters has on the equilibrium investment in the company under $P$ and the impact it has on the subgame perfect equilibrium level of investment in the company.

Corollary 5 The subgame perfect equilibrium level of investment in the company does not change as a result of a change in parameters if the equilibrium investment in the company under $P$ does not change.

Proof. The result follows from the fact that the proportional rule is the only rule that is always a part of the subgame perfect Nash equilibrium. Hence, if the equilibrium investment in the company under $P$ does not change as a result of a change in parameters, the subgame perfect equilibrium level of investment in the company does not change.

This corollary implies that if the equilibrium investment in the company under $C E A$ or $C E L$ drops as a result of a change in some parameter, this does not necessarily mean that the subgame perfect equilibrium level of investment in the company drops, since $P$ might still be leading to $n_{l} w_{l}+n_{h} w_{h}$ investment in the company. For example, as a result of a change in some parameter the investment in the company 
under $C E A(C E L)$ might drop from $n_{l} w_{l}+n_{h} w_{h}$ to $n_{l} w_{l}\left(n_{h} w_{h}\right)$, but under $P$ it might be still $n_{l} w_{l}+n_{h} w_{h}$. In that case, the subgame perfect equilibrium investment in the company is still $n_{l} w_{l}+n_{h} w_{h}$. The equilibrium investment in the company drops only if $\pi_{s} \geq \frac{\bar{r}-r_{f}}{r-r_{f}}$ changes to $\pi_{s}<\frac{\bar{r}-r_{f}}{r-r_{f}}$ as a result of a change in some parameter. Hence, there are still two possible equilibrium investment levels: $n_{l} w_{l}+n_{h} w_{h}$ and 0 . Similarly, the equilibrium investment may not rise as a result of a change in some parameter, even if it causes an increase in the investment under $C E A$ or $C E L$.

\section{Discussion}

In this section, we discuss our assumptions, the way we set up our model, our results, extension ideas and possible future research. For instance, one might notice that the return rate determination is exogenous in our model. Moreover, we assume identical risk-attitudes (i.e., risk neutrality) for all agents. Obviously, these are simplifying assumptions. Our main purpose in this research is to integrate the models of bankruptcy problem into a noncooperative game theoretical model that involves strategic decision-making under uncertainty. By endogenizing agents' decisions, we derive a noncooperative support for the proportional rule. Embedding the simple bankruptcy model into a strategic context requires some simplifications such as the ones we mentioned above. Below, we discuss what would happen if we did not make some of the assumptions.

Nonexistence of Pure Strategy Nash Equilibrium Under Different Risk Attitudes: If we assume that the high type agents are risk-neutral and the low type agents are risk-averse, we show that a pure strategy Nash Equilibrium might not exist under some parameter values. Below, we explain the dynamics of the non-existence result in more detail.

The reason of non-existence under different risk attitudes (in particular, risk neutral type $h$ agents and risk averse type $l$ agents) is the high degree of conflict of interest and the availability of an escape option (i.e., the risk-free asset) causing cyclicity. When we analyze the parameter conditions under which there is no pure strategy equilibrium, we see that the cost that is imposed on type $h$ agents (type $l$ agents) under $C E A(C E L)$ can be understood as the level of conflict of interest. For example, under $C E A$, if $c_{h}>\lambda_{c e a}>c_{l}$, then the term $\frac{n_{l}}{n_{h}}\left(r_{f}-r\right) w_{l}$ shows per-type $h$-capita transfer to type $l$ agents. A quick look at the term shows that if $n_{h}$ is high, then per-type $h$-capita transfer is low, if $n_{l}$ is high it is high and if $\left(r_{f}-r\right)$ is high, it is again high. Therefore, for some parameter values, under $C E A$, type $h$ agents do not play in against type $l$ agents playing $i n$. Hence, their best response to type $l$ agents playing in is to play out. And type $l$ agents' best response to type $h$ agents playing out is also out. However, type $h$ agents' best response to type $l$ agents playing out is to play in and type $l$ agents' best response to type $h$ agents playing in is to play $i n$. Hence, the process does not stop at an equilibrium. In the following, we construct an example in which there is no pure strategy Nash equilibrium under $C E A$. 
Example 2 Assume that $n_{l}=n_{h}=1, w_{l}=10, w_{h}=100, r=0.8, r_{f}=0.2$, and $\bar{r}=0.61$. Moreover, recall that type $h$ agent is risk neutral whereas type $l$ agent is risk-averse. This implies that type $l$ agent rejects fair gambles. Then, we can simply calculate the expected payoffs for the type $h$ agent as

$$
\begin{aligned}
P_{h}^{e} & =(0.7)(1+0.8) 100+(0.3)(1+0.2) 100=162 \text { and } \\
C E A_{h}^{e} & =(0.7)(1+0.8) 100+(0.3) 114=160.2 .
\end{aligned}
$$

Denote the utility function of the type $l$ agent as $U($.$) . Since$

$$
\begin{aligned}
V_{h, \text { out }} & =161, \\
V_{l, \text { out }} & =16.1, \text { and } \\
C E A_{l} & =18
\end{aligned}
$$

the payoff matrix can be written as

\begin{tabular}{|c|c|c|}
\hline$h \backslash l$ & in & out \\
\hline in & $160.2, U(18)$ & $162, U(16.1)$ \\
\hline out & $161,(0.7) U(18)+(0.3) U(12)$ & $161, U(16.1)$ \\
\hline
\end{tabular}

Risk-aversion implies that $(0.7) U(18)+(0.3) U(12)<U(16.2)$. If $(0.7) U(18)+$ $(0.3) U(12)<U(16.1)$ is also valid, then we do not have pure strategy Nash equilibrium in this game. $B R_{h}($ in $)=$ out, $B R_{l}($ out $)=$ out, $B R_{h}($ out $)=i n$ and $B R_{l}($ in $)=$ $i n$. Hence, there is a cycle. As the reader might realize, the statement " $P_{h}^{e} \geq V_{h, \text { out }}$ if and only if $U\left(P_{l}\right) \geq V_{l, o u t}$ " is not valid anymore, which causes the cycle. The nonexistence results Kibris \& Kibris (2008) have under $C E A$ in a similar setup confirms our expectations.

Nonexistence of Pure Strategy Nash Equilibrium Under Different Outside Asset Payoffs: We assumed that the risk-free asset brings the same payoff to both types of agents in case they invest in it. This is an unbiased (neutral) assumption to make. However there might be real life instances in which there are different risk-free assets available to different types of agents (e.g., the risk-free asset available to $h$ agents might pay higher (lower) than the risk-free asset available to type $l$ agents). In that case, the company's decision in equilibrium might be different than the one we described above. However, a problem with this more general approach is that, again, it can bring non-existence results for some parameter values. Hence, we kept our neutral assumption that risk-free asset pays the same return rate to all agents. In the following, we construct an example where there is no pure strategy Nash equilibrium in the subgame under $C E A$.

Example 3 Assume that $n_{l}=n_{h}=1, w_{l}=10, w_{h}=100, r=0.8, r_{f}=0.2$, $\bar{r}_{h}=0.61$ and $\bar{r}_{l}=0.63$. Then, we can simply calculate the expected payoffs as

$$
\begin{aligned}
P_{h}^{e} & =(0.7)(1+0.8) 100+(0.3)(1+0.2) 100=162, \\
C E A_{h}^{e} & =(0.7)(1+0.8) 100+(0.3) 114=160.2, \\
P_{l}^{e} & =(0.7)(1+0.8) 10+(0.3)(1+0.2) 10=16.2, \text { and } \\
C E A_{l}^{e} & =(0.7)(1+0.8) 10+(0.3) 18=18 .
\end{aligned}
$$


Risk-free asset brings payoffs $V_{h, \text { out }}=161$ and $V_{l, \text { out }}=16.3$. Hence, the payoff matrix can be written as

\begin{tabular}{|c|c|c|}
\hline$h \backslash l$ & in & out \\
\hline in & $160.2,18$ & $162,16.3$ \\
\hline out & $161,16.2$ & $161,16.3$ \\
\hline
\end{tabular}

As the reader can notice, type $h$ agent does not play in against type $l$ agents playing $i n$. Hence, his best response to type $l$ agent playing in is to play out. And type $l$ agent's best response to type $h$ agent playing out is also out. However, type $h$ agent's best response to type $l$ agent playing out is to play in and type $l$ agent's best response to type $h$ agent playing in is to play $i n$. Hence, the process does not stop at an equilibrium. As the reader might realize, it is again the invalidity of the statement " $P_{h}^{e} \geq V_{h, \text { out }}$ if and only if $P_{l}^{e} \geq V_{l, \text { out }}$ ", which causes the cycle.

Individual Optimization on the Level of Investment: In this paper, we assume that individuals do not maximize their expected payoffs by choosing the amount of money they want to invest in the company and in the savings account. They either invest in the company or deposit their money in a savings account. However, if we allowed them to optimize under risk-neutrality assumption, we would have corner solutions where each agent, again, will either fully invest in the company or in the savings account. Hence, our assumption, ex-post, is not very restrictive under risk-neutrality.

Below, we discuss some other aspects of our model such as distributive properties of the bankruptcy rules in bankruptcy problems with endogenous estates and possible extensions.

Distributive Properties: In a follow-up paper (see Karagozoglu, 2008), we analyze some distributive properties such as minimal rights first, securement of initial investments, initial investments first, reasonable lower bounds on awards, and reasonable lower bounds on losses in the class of bankruptcy problems with endogenous estates. We show that the proportional rule receives more support compared to the constrained equal awards and the constrained equal losses rules. Some normative properties that were not satisfied by the proportional rule in the general class of bankruptcy problems are satisfied by this rule in the class of bankruptcy problems with an endogenous estate. These changes are mostly due to the fact that estate and claims are not independent in this class. As a result, certain values of the estate for which the proportional rule does not satisfy certain properties are eliminated in this class of bankruptcy problems.

Bankruptcy Laws and Sequential Priority Rules: We observe that many bankruptcy laws apply certain sequential priority rules in the liquidation of bankrupt companies' assets. Priority classes are defined on the basis of seniority (e.g., founding stockholders or new stockholders), status of creditors (e.g., stockholder or bondholder) or status of claims (e.g., secured or unsecured) etc. The American bankruptcy law is a 
frequently quoted example in which priority classes are federal government (taxes), trustees (administrative expenses of the trustee) and two kinds of creditors (secured and unsecured claims). These rules and the constrained equal awards and constrained equal losses rules have a commonality: in all of these rules, there are people favored by the rule (i.e., receiving transfers) and there are people disfavored by the rule (i.e., making transfers). In this paper, we show that the strength of the proportional rule stems from its "neutrality" (zero transfers as a benchmark). Therefore, if we slightly modify our model to capture priority criteria such as seniority or status, there will, again, exist some parameter values for which, some agents do not invest in the company in equilibrium under the sequential priority rules. Loosely speaking, the proportional rule will outperform sequential priority rules in attracting investment to the company. Moreover, an important fact, which is in conformity with our results is that the proportional rule is applied in the liquidation process within each priority class in sequential priority rules.

Extension to $T>2$ Types: Extension of our results to $T>2$ types is a work in progress. We have some preliminary results, but this extension is not trivial at all since proving the comparative static results and the symmetry result is more complex than in the case of 2 types. The reason is that in the case of $T>2$ types, a change in the number type $t \in\{1,2, \ldots, T\}$ agents can cause an identity switch for some other types (some types might become transfer-makers and/or some other types might become transfer-receivers).

Competition Among Multiple Companies: In most cases, there are more than one company competing for the same group of investors in real life. In a follow up paper, we plan to introduce competition into the model analyzed here. This makes our model more realistic and also enable us to check the robustness of the results obtained in this paper. Preliminary results show that the income distribution has a greater impact on equilibrium than it has in this paper.

Increasing Returns and/or Decreasing Risk: In certain real-life circumstances, one might suggest that an increase in the investment volume can lead to an increase in the return rate (e.g., the investment project involves increasing returns to capital) and/or a decrease in the rate of risk (e.g., a higher level of capital increases the likelihood of success). If we incorporate these two possibilities, we expect that our results would quantitatively change. The relative (with respect to the proportional rule) positions of the constrained equal awards and the constrained equal losses rules would improve. However, we expect no qualitative change in the results, i.e., the proportional rule would still have an advantage over others.

\section{Conclusion}

In this paper, we introduced a new class of bankruptcy problems, which have an empirical appeal. In these bankruptcy problems, the value of the estate to be allocated to agents is endogenous and depends on agents' investment decisions. This is 
what we observe in many real life cases. For instance the amount to be allocated by a firm to its shareholders/stockholders may depend on the initial amount of money borrowed from them. Moreover, in line with some recent suggestions in favor of a more liberal bankruptcy law, which provides a menu of bankruptcy procedures and allows companies to select among them (see Hart, 2000), we allow the company in our model to choose from a menu of bankruptcy rules that consists of three wellknown rules, i.e., the proportional rule, the constrained equal awards rule and the constrained equal losses rule. Company's objective in choosing a bankruptcy rule is to maximize the investment volume, which would lead to maximum profit. Agents observe the choice made by the company and decide whether to lend money to the company (risky investment) or deposit their money in a savings account (risk-free investment).

Our results show that the proportional rule receives a strong and robust support in this setting. There always exists a subgame perfect Nash equilibrium, which involves the proportional rule. A direct implication is that there is no equilibrium in which the proportional rule leads to a lower level of investment volume than the other rules. This result is independent of the income distribution in the society and holds even under one-sided uncertainty on income distribution (i.e., the company does not know the income distribution perfectly). In fact, for probability distributions that assign non-zero probability to all possible income distributions, the proportional rule would be the unique optimal strategy for an expected payoff maximizing company. We also extend the menu of rules to include a subset of the TAL-family and show that results do not change a lot. This is an important extension since the TAL-family contains infinitely many rules some of which are the Talmud rule, the constrained equal awards rule and the constrained equal losses rule. Our results provide, at least, a partial explanation from a strategic point of view for the fact that the proportional rule is frequently used in the liquidation process of a bankrupt company's assets (e.g., Hart, 2000 and Kaminski, 2006). Finally, we also provide a noncooperative justification for the normative idea proposed by the equity theory of Selten (1978). To the best of our knowledge, this is the first paper which models the bankruptcy rule determination as a sequential game between lender (company) and borrowers (agents). It is also the first paper, which embeds the classical bankruptcy problem in a decision-making under uncertainty environment. 


\section{References}

[1] Araujo, A. P. \& Páscoa, M. R. (2002) "Bankruptcy in a model of unsecured claims", Economic Theory, 20(3), pp. 455 - 481.

[2] Aumann, R. \& Maschler, M. (1985) "Game theoretic analysis of a bankruptcy problem from the Talmud", Journal of Economic Theory, 36(2), pp. 195 $-213$.

[3] Bergantiños, G. \& Sanchez, E. (2002) "The proportional rule for problems with constraints and claims", Mathematical Social Sciences, 43(2), pp. 225 - 249.

[4] Chambers, C. P. \& Thomson, W. (2002) "Group order preservation and the proportional rule for the adjudication of conflicting claims", Mathematical Social Sciences, 44(3), pp. 235 - 252.

[5] Chang, C. \& Hu, C-C. (2008) "A non-cooperative interpretation of the $f$ just rules of bankruptcy problems", Games and Economic Behavior, 63(1), pp. $133-144$.

[6] Ching, S. \& Kakkar, V. (2001) "A market approach to the bankruptcy problem", mimeo, City University of Hong Kong.

[7] Chun, Y. (1988) "The proportional solution for rights problem", Mathematical Social Sciences, 15(3), pp. 231 - 246.

[8] Chun, Y. (1989) "A noncooperative justification for egalitarian surplus sharing", Mathematical Social Sciences, 17(3), pp. 245 - 261.

[9] Chun, Y. \& Lee, J. (2007) "On the convergence of the random arrival rule in large claims problems", International Journal of Game Theory, 36(2), pp. 259 273.

[10] Curiel, I., Maschler, M. \& Tijs, S. H. (1987) "Bankruptcy games", Zeitschrift fur Operations Research, 31(5), pp. A143 - A159.

[11] Dagan, N. \& Volij, O. (1993) "The bankruptcy problem: a cooperative bargaining approach", Mathematical Social Sciences, 26(3), pp. 287 - 297.

[12] Dagan, N., Serrano, R. \& Volij, O. (1997) "A noncooperative view of consistent bankruptcy rules", Games and Economic Behavior, 18(1), pp. 55 72 .

[13] De Frutos, M. A. (1999) "Coalitional manipulations in a bankruptcy problem", Review of Economic Design, 4(3), pp. 255 - 272.

[14] Gächter, S. \& Riedl, A. (2005) "Moral property rights in bargaining with infeasible claims", Management Science, 51(2), pp. 249 - 263. 
[15] Gächter, S. \& Riedl, A. (2006) "Dividing justly in bargaining problems with claims", Social Choice and Welfare, 27(3), pp. 571 - 594.

[16] García-Jurado, I., González-Díaz, J. \& Villar, A. (2006) "A noncooperative approach to bankruptcy problems", Spanish Economic Review, 8(3), pp. $189-197$.

[17] Hart, O. (2000) "Different approaches to bankruptcy", NBER Working Paper \# 7921.

[18] Herrero, C. (2003) "Equal awards vs. equal losses: duality in bankruptcy", In: Sertel MR, Koray S (eds.) Adv Econ Des. Springer, Berlin Heidelberg New York.

[19] Herrero, C., Moreno-Ternero, J. D. \& Ponti, G. (2006) "On the adjudication of conflicting claims: an experimental study", CORE Discussion Paper $2006 / 62$.

[20] Herrero, C. \& Villar, A. (2001) "The three musketeers: four classical solutions to bankruptcy problems", Mathematical Social Sciences, 42(3), pp. 307 328.

[21] Hougaard, J. L. \& Østerdal, L. P. (2005) "Inequality preserving rationing", Economics Letters, 87(3), pp. 355 - 360.

[22] Ju, B-G. (2003) "Manipulation via merging and splitting in claims problems", Review of Economic Design, 8(2), pp. 205 - 215.

[23] Ju, B-G., Miyagawa, E. \& Sakai, T. (2007) "Non-manipulable division rules in claims problems and generalizations", Journal of Economic Theory, 132(1), pp. 1 - 26.

[24] Kaminski, M. M. (2006) "Parametric rationing methods", Games and Economic Behavior, 54(1), pp. 115 - 133.

[25] Karagozoglu, E. (2008) "Distributive concerns in the bankruptcy problem with an endogenous estate", mimeo, Maastricht University.

[26] Kibris, O. \& Kibris, A. (2008) "On the investment implications of bankruptcy laws", mimeo, Sabanci University.

[27] Moreno-Ternero, J. D. (2002) "Noncooperative support for the proportional rule in bankruptcy problems", mimeo, Universidad de Alicante.

[28] Moreno-Ternero, J. D. (2006a) "Proportionality and non-manipulability in bankruptcy problems", International Game Theory Review, 8(1), pp. 127 - 139.

[29] Moreno-Ternero, J. D. (2006b) "Proportional rule for multi-issue bankruptcy problems", CORE Discussion Paper \# 2006/76. 
[30] Moreno-Ternero, J. D. \& Villar, A. (2006) "The TAL-family of rules for bankruptcy problems", Social Choice and Welfare, 27(2), pp. 231 - 249.

[31] Moulin, H. (2000) "Priority rules and other asymmetric rationing methods," Econometrica, 68(3), pp. 643 - 684.

[32] Moulin, H. (2002) "Axiomatic cost and surplus sharing" in: Arrow K., Sen A., Suzumura K. (eds.) Handbook of Social Choice and Welfare, vol. 1. Elsevier, Amsterdam.

[33] O‘Neill, B. (1982) "A problem of rights arbitration from the Talmud", Mathematical Social Sciences, 2(4), pp. 345 - 371.

[34] Selten, R. (1978) "The equity principle in economic behavior", in Gottinger H.W. \& Leinfellner W. (eds.) Decision Theory and Social Effects. Kluwer, Dordrecht, pp. $289-301$.

[35] Thomson, W. (2003) "Axiomatic and game-theoretic analysis of bankruptcy and taxation problems: a survey", Mathematical Social Sciences, 45(3), pp. 249 $-297$.

[36] Thomson, W. (2006) How to Divide When There isn't Enough; From the Talmud to Game Theory, unpublished manuscript.

[37] Young, P. (1985) "Cost Allocation" in: Young P. (eds.) Fair Allocation. Proceedings of Symposia in Applied Mathematics, vol. 33, Providence RI: The American Mathematical Society.

[38] Yaari, M. E. \& Bar-Hillel, M. (1984) "On dividing justly", Social Choice and Welfare, 1(1), pp. 1 - 24. 


\section{Appendix:}

\section{A - Comparative Static Analysis Calculations}

\section{Closed Form Expressions for Transfers}

$$
\begin{aligned}
S_{h}(C E A, i n) & =\frac{n_{l}\left(r_{f}-r\right) w_{l}}{n_{h}} \text { if } c_{h}>\lambda_{\text {cea }} \geq c_{l} \\
S_{h}(C E A, \text { in }) & =\frac{n_{l}}{n_{h}+n_{l}}\left(1+r_{f}\right)\left(w_{l}-w_{h}\right) \text { if } c_{h}>c_{l} \geq \lambda_{c e a} \\
S_{l}(C E A, i n) & =\left(r-r_{f}\right) w_{l} \text { if } c_{h}>\lambda_{c e a} \geq c_{l} \\
S_{l}(C E A, i n) & =\frac{n_{l}}{n_{h}+n_{l}}\left(1+r_{f}\right)\left(w_{h}-w_{l}\right) \text { if } c_{h}>c_{l} \geq \lambda_{c e a} \\
S_{l}(C E L, i n) & =\frac{n_{h}}{n_{h}+n_{l}}\left(r-r_{f}\right)\left(w_{l}-w_{h}\right) \text { if } c_{h}>c_{l}>\lambda_{c e l} \\
S_{l}(C E L, i n) & =-\left(1+r_{f}\right) w_{l} \text { if } c_{h}>\lambda_{c e l} \geq c_{l} \\
S_{h}(C E L, i n) & =\frac{n_{l}}{n_{h}+n_{l}}\left(r-r_{f}\right)\left(w_{h}-w_{l}\right) \text { if } c_{h}>c_{l}>\lambda_{c e l} \\
S_{h}(C E L, i n) & =\frac{n_{l}}{n_{h}}\left(1+r_{f}\right) w_{l} \text { if } c_{h}>\lambda_{c e l} \geq c_{l}
\end{aligned}
$$

\section{Comparative Static Analysis Calculations}

Since $n_{h, \text { in }}$ and $n_{l, i n}$ are discrete variables, we cannot look at derivatives. Hence, we look at differences. Now, we define the difference operator. The change in $S_{t}(., .,$. with respect to a unit change in $n_{t, i n}$ is denoted by $\frac{\triangle S_{t}\left(F, n_{h, i n}, n_{l, i n}\right)}{\triangle n_{t, i n}}$.

$$
S_{h}(C E A, \text { in })=\frac{n_{l}\left(r_{f}-r\right) w_{l}}{n_{h}} \text { if } c_{h}>\lambda_{c e a} \geq c_{l}
$$

- Change in $S_{h}(C E A, i n)$ with respect to $n_{l}$, if $c_{h}>\lambda_{c e a} \geq c_{l}$

$$
\frac{\triangle S_{h}(C E A, i n)}{\triangle n_{l}}=\frac{w_{l}\left(r_{f}-r\right)}{n_{h}}<0
$$

- Change in $S_{h}(C E A, i n)$ with respect to $n_{h}$, if $c_{h}>\lambda_{c e a} \geq c_{l}$

$$
\frac{\triangle S_{h}(C E A, i n)}{\triangle n_{h}}=\frac{-n_{l} w_{l}\left(r_{f}-r\right)}{\left(n_{h}\right)^{2}} \text { or } \frac{-n_{l} w_{l}\left(r_{f}-r\right)}{\left(n_{h}+1\right) n_{h}}>0
$$

- Change in $S_{h}(C E A, i n)$ with respect to $w_{l}$, if $c_{h}>\lambda_{c e a} \geq c_{l}$

$$
\frac{\partial S_{h}(C E A, i n)}{\partial w_{l}}=\frac{n_{l}\left(r_{f}-r\right)}{n_{h}}<0
$$

- Change in $S_{h}(C E A, i n)$ with respect to $w_{h}$, if $c_{h}>\lambda_{c e a} \geq c_{l}$ 
$\frac{\partial S_{h}(C E A, i n)}{\partial w_{h}}=0$

$$
S_{h}(C E A, i n)=\frac{n_{l}}{n_{h}+n_{l}}\left(1+r_{f}\right)\left(w_{l}-w_{h}\right) \text { if } c_{h}>c_{l} \geq \lambda_{c e a}
$$

- Change in $S_{h}(C E A, i n)$ with respect to $n_{l}$, if $c_{h}>c_{l} \geq \lambda_{c e a}$

$$
\frac{\triangle S_{h}(C E A, i n)}{\triangle n_{l}}=\frac{n_{h}\left(1+r_{f}\right)\left(w_{l}-w_{h}\right)}{\left(n_{h}+n_{l}\right)^{2}} \text { or } \frac{n_{h}\left(1+r_{f}\right)\left(w_{l}-w_{h}\right)}{\left(n_{h}+n_{l}+1\right)\left(n_{h}+n_{l}\right)}<0
$$

- Change in $S_{h}(C E A, i n)$ with respect to $n_{h}$, if $c_{h}>c_{l} \geq \lambda_{c e a}$

$$
\frac{\triangle S_{h}(C E A, i n)}{\triangle n_{h}}=-\frac{n_{l}\left(1+r_{f}\right)\left(w_{l}-w_{h}\right)}{\left(n_{h}+n_{l}\right)^{2}} \text { or }-\frac{n_{l}\left(1+r_{f}\right)\left(w_{l}-w_{h}\right)}{\left(n_{h}+n_{l}+1\right)\left(n_{h}+n_{l}\right)}>0
$$

- Change in $S_{h}(C E A, i n)$ with respect to $w_{l}$, if $c_{h}>c_{l} \geq \lambda_{c e a}$ $\frac{\partial S_{h}(C E A, i n)}{\partial w_{l}}=\frac{n_{l}}{n_{h}+n_{l}}\left(1+r_{f}\right)>0$

- Change in $S_{h}(C E A$, in $)$ with respect to $w_{h}$, if $c_{h}>c_{l} \geq \lambda_{c e a}$

$$
\begin{aligned}
\frac{\partial S_{h}(C E A, i n)}{\partial w_{h}}=-\frac{n_{l}}{n_{h}+n_{l}}\left(1+r_{f}\right) & <0 \\
S_{l}(C E A, i n) & =\left(r-r_{f}\right) w_{l} \text { if } c_{h}>\lambda_{c e a} \geq c_{l}
\end{aligned}
$$

- Change in $S_{l}(C E A$, in $)$ with respect to $n_{l}$, if $c_{h}>\lambda_{c e a} \geq c_{l}$ $\frac{\triangle S_{l}(C E A, i n)}{\triangle n_{l}}=0$

- Change in $S_{l}(C E A, i n)$ with respect to $n_{h}$, if $c_{h}>\lambda_{c e a} \geq c_{l}$ $\frac{\triangle S_{l}(C E A, i n)}{\triangle n_{h}}=0$

- Change in $S_{l}(C E A, i n)$ with respect to $w_{l}$, if $c_{h}>\lambda_{c e a} \geq c_{l}$ $\frac{\partial S_{l}(C E A, i n)}{\partial w_{l}}=\left(r-r_{f}\right)>0$

- Change in $S_{l}(C E A, i n)$ with respect to $w_{h}$, if $c_{h}>\lambda_{c e a} \geq c_{l}$

$$
\begin{aligned}
& \frac{\partial S_{l}(C E A, i n)}{\partial w_{h}}=0 \\
& \quad S_{l}(C E A, i n)=\frac{n_{h}}{n_{h}+n_{l}}\left(1+r_{f}\right)\left(w_{h}-w_{l}\right) \text { if } c_{h}>c_{l} \geq \lambda_{c e a}
\end{aligned}
$$

- Change in $S_{l}(C E A, i n)$ with respect to $n_{l}$, if $c_{h}>c_{l} \geq \lambda_{c e a}$

$$
\frac{\triangle S_{l}(C E A, i n)}{\triangle n_{l}}=-\frac{n_{h}\left(1+r_{f}\right)\left(w_{h}-w_{l}\right)}{\left(n_{h}+n_{l}\right)^{2}} \text { or }-\frac{n_{h}\left(1+r_{f}\right)\left(w_{h}-w_{l}\right)}{\left(n_{h}+n_{l}+1\right)\left(n_{h}+n_{l}\right)}<0
$$

- Change in $S_{l}(C E A, i n)$ with respect to $n_{h}$, if $c_{h}>c_{l} \geq \lambda_{c e a}$ 
$\frac{\triangle S_{l}(C E A, i n)}{\triangle n_{h}}=\frac{n_{l}\left(1+r_{f}\right)\left(w_{h}-w_{l}\right)}{\left(n_{h}+n_{l}\right)^{2}}$ or $\frac{n_{l}\left(1+r_{f}\right)\left(w_{h}-w_{l}\right)}{\left(n_{h}+n_{l}+1\right)\left(n_{h}+n_{l}\right)}>0$

- Change in $S_{l}(C E A$, in $)$ with respect to $w_{l}$, if $c_{h}>c_{l} \geq \lambda_{c e a}$ $\frac{\partial S_{l}(C E A, i n)}{\partial w_{l}}=-\frac{n_{h}}{n_{h}+n_{l}}\left(1+r_{f}\right)<0$

- Change in $S_{l}(C E A, i n)$ with respect to $w_{h}$, if $c_{h}>c_{l} \geq \lambda_{c e a}$ $\frac{\partial S_{l}(C E A, i n)}{\partial w_{h}}=\frac{n_{h}}{n_{h}+n_{l}}\left(1+r_{f}\right)>0$

$$
S_{l}(C E L, i n)=\frac{n_{h}}{n_{h}+n_{l}}\left(r-r_{f}\right)\left(w_{l}-w_{h}\right) \text { if } c_{h}>c_{l}>\lambda_{c e l}
$$

- Change in $S_{l}(C E L, i n)$ with respect to $n_{l}$, if $c_{h}>c_{l}>\lambda_{c e l}$ $\frac{\triangle S_{l}(C E L, i n)}{\triangle n_{l}}=-\frac{n_{h}\left(w_{l}-w_{h}\right)\left(r-r_{f}\right)}{\left(n_{h}+n_{l}\right)^{2}}$ or $-\frac{n_{h}\left(w_{l}-w_{h}\right)\left(r-r_{f}\right)}{\left(n_{h}+n_{l}+1\right)\left(n_{h}+n_{l}\right)}>0$

- Change in $S_{l}(C E L, i n)$ with respect to $n_{h}$, if $c_{h}>c_{l}>\lambda_{c e l}$ $\frac{\triangle S_{l}(C E L, i n)}{\triangle n_{h}}=\frac{n_{l}\left(w_{l}-w_{h}\right)\left(r-r_{f}\right)}{\left(n_{h}+n_{l}\right)^{2}}$ or $\frac{n_{l}\left(w_{l}-w_{h}\right)\left(r-r_{f}\right)}{\left(n_{h}+n_{l}+1\right)\left(n_{h}+n_{l}\right)}<0$

- Change in $S_{l}(C E L, i n)$ with respect to $w_{l}$, if $c_{h}>c_{l}>\lambda_{c e l}$ $\frac{\partial S_{l}(C E L, i n)}{\partial w_{l}}=\frac{n_{h}}{n_{h}+n_{l}}\left(r-r_{f}\right)>0$

- Change in $S_{l}(C E L, i n)$ with respect to $w_{h}$, if $c_{h}>c_{l}>\lambda_{c e l}$ $\frac{\partial S_{l}(C E L, i n)}{\partial w_{h}}=-\frac{n_{h}}{n_{h}+n_{l}}\left(r-r_{f}\right)<0$

$$
S_{l}(C E L, i n)=-\left(1+r_{f}\right) w_{l} \text { if } c_{h}>\lambda_{c e l} \geq c_{l}
$$

- Change in $S_{l}(C E L, i n)$ with respect to $n_{l}$, if $c_{h}>\lambda_{c e l} \geq c_{l}$ $\frac{\triangle S_{l}(C E L, i n)}{\triangle n_{l}}=0$

- Change in $S_{l}(C E L, i n)$ with respect to $n_{h}$, if $c_{h}>\lambda_{c e l} \geq c_{l}$ $\frac{\triangle S_{l}(C E L, i n)}{\triangle n_{h}}=0$

- Change in $S_{l}(C E L, i n)$ with respect to $w_{l}$, if $c_{h}>\lambda_{c e l} \geq c_{l}$ $\frac{\partial S_{l}(C E L, i n)}{\partial w_{l}}=-\left(1+r_{f}\right)<0$

- Change in $S_{l}(C E L, i n)$ with respect to $w_{h}$, if $c_{h}>\lambda_{c e l} \geq c_{l}$ $\frac{\partial S_{l}(C E L, i n)}{\partial w_{h}}=0$

$$
S_{h}(C E L, i n)=\frac{n_{l}}{n_{h}+n_{l}}\left(r-r_{f}\right)\left(w_{h}-w_{l}\right) \text { if } c_{h}>c_{l}>\lambda_{c e l}
$$


- Change in $S_{h}(C E L, i n)$ with respect to $n_{l}$, if $c_{h}>c_{l}>\lambda_{c e l}$

$$
\frac{\triangle S_{h}(C E L, i n)}{\triangle n_{l}}=\frac{n_{h}\left(w_{h}-w_{l}\right)\left(r-r_{f}\right)}{\left(n_{h}+n_{l}\right)^{2}} \text { or } \frac{n_{h}\left(w_{h}-w_{l}\right)\left(r-r_{f}\right)}{\left(n_{h}+n_{l}+1\right)\left(n_{h}+n_{l}\right)}>0
$$

- Change in $S_{h}(C E L, i n)$ with respect to $n_{h}$, if $c_{h}>c_{l}>\lambda_{c e l}$

$$
\frac{\triangle S_{h}(C E L, i n)}{\triangle n_{h}}=-\frac{n_{l}\left(w_{h}-w_{l}\right)\left(r-r_{f}\right)}{\left(n_{h}+n_{l}\right)^{2}} \text { or }-\frac{n_{l}\left(w_{h}-w_{l}\right)\left(r-r_{f}\right)}{\left(n_{h}+n_{l}+1\right)\left(n_{h}+n_{l}\right)}<0
$$

- Change in $S_{h}(C E L, i n)$ with respect to $w_{l}$, if $c_{h}>c_{l}>\lambda_{c e l}$

$$
\frac{\partial S_{h}(C E L, i n)}{\partial w_{l}}=-\frac{n_{l}}{n_{h}+n_{l}}\left(r-r_{f}\right)<0
$$

- Change in $S_{h}(C E L, i n)$ with respect to $w_{h}$, if $c_{h}>c_{l}>\lambda_{c e l}$

$$
\begin{aligned}
\frac{\partial S_{h}(C E L, i n)}{\partial w_{h}}=\frac{n_{l}}{n_{h}+n_{l}}\left(r-r_{f}\right) & >0 \\
S_{h}(C E L, i n) & =\frac{n_{l}}{n_{h}}\left(1+r_{f}\right) w_{l} \text { if } c_{h}>\lambda_{c e l} \geq c_{l}
\end{aligned}
$$

- Change in $S_{h}(C E L, i n)$ with respect to $n_{l}$, if $c_{h}>\lambda_{c e l} \geq c_{l}$

$$
\frac{\triangle S_{h}(C E L, i n)}{\triangle n_{l}}=\frac{\left(1+r_{f}\right) w_{l}}{n_{h}}>0
$$

- Change in $S_{h}(C E L, i n)$ with respect to $n_{h}$, if $c_{h}>\lambda_{c e l} \geq c_{l}$

$$
\frac{\triangle S_{h}(C E L, i n)}{\triangle n_{h}}=-\frac{n_{l}\left(1+r_{f}\right) w_{l}}{\left(n_{h}\right)^{2}} \text { or }-\frac{n_{l}\left(1+r_{f}\right) w_{l}}{\left(n_{h}+1\right)\left(n_{h}\right)}<0
$$

- Change in $S_{h}(C E L, i n)$ with respect to $w_{l}$, if $c_{h}>\lambda_{c e l} \geq c_{l}$

$$
\frac{\partial S_{h}(C E L, i n)}{\partial w_{l}}=\frac{n_{l}}{n_{h}}\left(1+r_{f}\right)>0
$$

- Change in $S_{h}(C E L, i n)$ with respect to $w_{h}$, if $c_{h}>\lambda_{c e l} \geq c_{l}$

$$
\frac{\partial S_{h}(C E L, i n)}{\partial w_{h}}=0
$$

\section{B - Proofs of Preparatory Results}

Proof of Lemma 1. (i) Assume that the number of claimants for whom $c_{j} \leq \lambda_{\text {cea }}$ is $k^{*}<n$. It immediately follows that for all $c_{j} \leq \lambda_{\text {cea }}, C E A_{j}(C, E)=c_{j}$. Therefore, for all $c_{j} \leq \lambda_{c e a}$, since $P_{j}(C, E)<c_{j}$, we get $C E A_{j}(C, E)>P_{j}(C, E)$. We now analyze the case, $c_{j}>\lambda_{c e a}$. For all such claimants, $C E A_{j}(C, E)=\lambda_{c e a}$. This can be written as

$$
C E A_{j}(C, E)=\lambda_{c e a}=\frac{E-\sum_{i=1}^{k^{*}} c_{i}}{n-k^{*}} .
$$

Furthermore, we know that

$$
P_{j}(C, E)=c_{j} \frac{E}{\sum_{i \in N} c_{i}} .
$$


To find out whether there exists $c^{*}$, which satisfies the statement in the lemma, we check the equality,

$$
\frac{E-\sum_{i=1}^{k^{*}} c_{i}}{n-k^{*}}=c^{*} \frac{E}{\sum_{i \in N} c_{i}}
$$

This can be rewritten as

$$
c^{*}=\frac{\left(E-\sum_{i=1}^{k^{*}} c_{i}\right) \sum_{i \in N} c_{i}}{\left(n-k^{*}\right) E}=\lambda_{c e a} \frac{\sum_{i \in N} c_{i}}{E} .
$$

Since $\left(E-\sum_{i=1}^{k^{*}} c_{i}\right)>0, c^{*}>0$. Moreover, since

$$
\frac{\sum_{i \in N} c_{i}}{E}>1
$$

(by the definition of bankruptcy), we have $c^{*}>\lambda_{\text {cea }}$. Now, pick an agent $j$ such that $\lambda_{\text {cea }}<c_{j}$. Then,

$$
c_{j} \lesseqgtr c^{*} \Leftrightarrow P_{j}(C, E)=c_{j} \frac{E}{\sum_{i \in N} c_{i}} \lesseqgtr c^{*} \frac{E}{\sum_{i \in N} c_{i}} .
$$

Using (12), we can rewrite this as

$$
c_{j} \lesseqgtr c^{*} \Leftrightarrow P_{j}(C, E)=c_{j} \frac{E}{\sum_{i \in N} c_{i}} \lesseqgtr \lambda_{c e a} \frac{\sum_{i \in N} c_{i}}{E} \frac{E}{\sum_{i \in N} c_{i}}=\lambda_{c e a}=C E A_{j}(C, E) .
$$

Thus, the result follows.

(ii) Assume that the number of claimants for whom $c_{j} \leq \lambda_{c e l}$ is $\widetilde{k}<n$. It immediately follows that for all $c_{j} \leq \lambda_{\text {cel }}, C E L_{j}(C, E)=0$. Therefore, for all $c_{j} \leq \lambda_{\text {cel }}$, since $0<P_{j}(C, E)$, we get $C E L_{j}(C, E)<P_{j}(C, E)$. We now analyze the case $c_{j}>\lambda_{c e l}$. For all such claimants, $C E L_{j}(C, E)=c_{j}-\lambda_{c e l}>0$, where $\lambda_{c e l}$ can be written as

$$
\lambda_{c e l}=\frac{\sum_{i=\widetilde{k}+1}^{n} c_{i}-E}{n-\widetilde{k}} .
$$

Therefore, for all $c_{j}>\lambda_{c e l}, C E L_{j}(C, E)$ can be written as

$$
C E L_{j}(C, E)=c_{j}-\frac{\sum_{i=\widetilde{k}+1}^{n} c_{i}-E}{n-\widetilde{k}} .
$$

Furthermore, we know that

$$
P_{j}(C, E)=c_{j} \frac{E}{\sum_{i \in N} c_{i}}
$$

To find out whether there exists $\widetilde{c}$, which satisfies the statement in the lemma, we check the equality,

$$
\widetilde{c}-\frac{\sum_{i=\widetilde{k}+1}^{n} c_{i}-E}{n-\widetilde{k}}=\widetilde{c} \frac{E}{\sum_{i \in N} c_{i}} .
$$


This can be rewritten as

$$
\widetilde{c}=\frac{\left(\sum_{i=\widetilde{k}+1}^{n} c_{i}-E\right) \sum_{i \in N} c_{i}}{(n-\widetilde{k})\left(\sum_{i \in N} c_{i}-E\right)}=\lambda_{c e l} \frac{\sum_{i \in N} c_{i}}{\sum_{i \in N} c_{i}-E}>0 .
$$

Moreover, since

$$
\frac{\sum_{i \in N} c_{i}}{\sum_{i \in N} c_{i}-E}>1
$$

we have $\widetilde{c}>\lambda_{\text {cel }}$. Now pick an agent $j$ such that $c_{j}>\lambda_{\text {cel }}$. Then,

$$
c_{j} \lesseqgtr \widetilde{c} \Leftrightarrow P_{j}(C, E)=c_{j} \frac{E}{\sum_{i \in N} c_{i}} \lesseqgtr \widetilde{c} \frac{E}{\sum_{i \in N} c_{i}} .
$$

Using (13), we can rewrite this as

$$
c_{j} \lesseqgtr \widetilde{c} \Leftrightarrow P_{j}(C, E)=c_{j} \frac{E}{\sum_{i \in N} c_{i}} \lesseqgtr \lambda_{c e l} \frac{\sum_{i \in N} c_{i}}{\sum_{i \in N} c_{i}-E} \frac{E}{\sum_{i \in N} c_{i}}=\lambda_{c e l} \frac{E}{\sum_{i \in N} c_{i}-E} .
$$

We know that an agent $j$ with a claim $c_{j}>\lambda_{\text {cel }}$ receives a payoff, $C E L_{j}(C, E)=$ $c_{j}-\lambda_{c e l}$ and at $c_{j}=\widetilde{c}$,

$$
C E L_{j}(C, E)=c_{j}-\lambda_{c e l}=\lambda_{c e l} \frac{E}{\sum_{i \in N} c_{i}-E}=P_{j}(C, E) .
$$

Moreover, at $c_{j}=\lambda_{c e l}$

$$
P_{j}(C, E)=\lambda_{c e l} \frac{E}{\sum_{i \in N} c_{i}}>0=C E L_{j}(C, E) .
$$

Therefore, these two linear functions (i.e., $P_{j}(C, E)=c_{j}\left(E / \sum_{i \in N} c_{i}\right)$ and $C E L_{j}(C, E)=$ $\left.c_{j}-\lambda_{c e l}\right)$ take different values at $c_{j}=\lambda_{c e l}$ and the same value at $c_{j}=\widetilde{c}$. In particular, $P_{j}(C, E)$ takes a higher value at $c_{j}=\lambda_{c e l}$. Therefore, $c_{j} \lesseqgtr \widetilde{c}$ implies

$$
P_{j}(C, E)=c_{j} \frac{E}{\sum_{i \in N} c_{i}} \gtreqless C E L_{j}(C, E) .
$$

Hence, $\widetilde{c}$ satisfies the statement in (ii).

Proof of Lemma 2. Under $C E A$, there are two cases: (a-1) $c_{h}>c_{l}>\lambda_{\text {cea }}$ and (a-2) $c_{h}>\lambda_{\text {cea }} \geq c_{l}$.

(a-1) If $c_{h}>c_{l} \geq \lambda_{c e a}$, then $C E A$ allocates the estate equally. Hence, $C E A_{l}(C, E)=$ $C E A_{h}(C, E)=\frac{E}{n_{h}+n_{l}}$. Therefore,

$$
S_{l}(E, C, C E A)=\left(\frac{E}{n}-\frac{E}{n_{h} c_{h}+n_{l} c_{l}} c_{l}\right),
$$

which satisfies

$$
S_{l}(E, C, C E A)=\frac{E n_{h}\left[c_{h}-c_{l}\right]}{\left[n_{h}+n_{l}\right]\left[n_{h} c_{h}+n_{l} c_{l}\right]}>0
$$


Similarly, $S_{h}(E, C, C E A)$ can be written as

$$
S_{h}(E, C, C E A)=\left(\frac{E}{n}-\frac{E}{n_{h} c_{h}+n_{l} c_{l}} c_{h}\right),
$$

which satisfies

$$
S_{h}(E, C, C E A)=\frac{E n_{l}\left[c_{l}-c_{h}\right]}{\left[n_{h}+n_{l}\right]\left[n_{h} c_{h}+n_{l} c_{l}\right]}<0 .
$$

(a-2) If $c_{h}>\lambda_{\text {cea }} \geq c_{l}$, then for each agent with claim $c_{l}, C E A_{l}(C, E)=c_{l}$ and for each agent with claim $c_{h}, C E A_{h}(C, E)=\frac{E-n_{l} c_{l}}{n_{h}}$. Therefore,

$$
S_{l}(E, C, C E A)=c_{l}\left(1-\frac{E}{n_{h} c_{h}+n_{l} c_{l}}\right)
$$

which satisfies

$$
S_{l}(E, C, C E A)=\frac{c_{l}\left[n_{h} c_{h}+n_{l} c_{l}-E\right]}{n_{h} c_{h}+n_{l} c_{l}}>0 .
$$

Similarly, $S_{h}(E, C, C E A)$ can be written as

$$
S_{h}(E, C, C E A)=\left(\frac{E-n_{l} c_{l}}{n_{h}}-\frac{E}{n_{h} c_{h}+n_{l} c_{l}}\right),
$$

which satisfies

$$
S_{h}(E, C, C E A)=\frac{n_{l} c_{l}\left[E-n_{h} c_{h}-n_{l} c_{l}\right]}{n_{h}\left[n_{h} c_{h}+n_{l} c_{l}\right]}<0 .
$$

Under $C E L$, there are two cases: (b-1) $c_{h}>c_{l}>\lambda_{\text {cel }}$ and (b-2) $c_{h}>\lambda_{c e l} \geq c_{l}$.

(b-1) If $c_{h}>c_{l}>\lambda_{c e l}$, then $C E L$ allocates losses equally. Hence, for each agent with claim $c_{l}, C E L_{l}(C, E)=c_{l}-\left(\frac{n_{h} c_{h}+n_{l} c_{l}-E}{n_{h}+n_{l}}\right)$ and for each agent with claim $c_{h}$, $C E L_{h}(C, E)=c_{h}-\left(\frac{n_{h} c_{h}+n_{l} c_{l}-E}{n_{h}+n_{l}}\right)$. Therefore,

$$
S_{l}(E, C, C E L)=\left[c_{l}-\left(\frac{n_{h} c_{h}+n_{l} c_{l}-E}{n_{h}+n_{l}}\right)-\frac{E}{n_{h} c_{h}+n_{l} c_{l}} c_{l}\right],
$$

which satisfies

$$
S_{l}(E, C, C E L)=\frac{n_{h}\left[c_{l}-c_{h}\right]\left[n_{h} c_{h}+n_{l} c_{l}-E\right]}{\left[n_{h}+n_{l}\right]\left[n_{h} c_{h}+n_{l} c_{l}\right]}<0 .
$$

Similarly, $S_{h}(E, C, C E L)$ can be written as

$$
S_{h}(E, C, C E L)=\left[c_{h}-\left(\frac{n_{h} c_{h}+n_{l} c_{l}-E}{n_{h}+n_{l}}\right)-\frac{E}{n_{h} c_{h}+n_{l} c_{l}} c_{h}\right],
$$

which satisfies

$$
S_{h}(E, C, C E L)=\frac{n_{l}\left[c_{h}-c_{l}\right]\left[n_{h} c_{h}+n_{l} c_{l}-E\right]}{\left[n_{h}+n_{l}\right]\left[n_{h} c_{h}+n_{l} c_{l}\right]}>0 .
$$


(b-2) If $c_{h}>\lambda_{c e l} \geq c_{l}$, then for each agent with claim $c_{l}, C E L_{l}(C, E)=0$ and for each agent with claim $c_{h}, C E L_{h}(C, E)=\frac{E}{n_{h}}$. Therefore,

$$
S_{l}(E, C, C E L)=-\frac{E}{n_{h} c_{h}+n_{l} c_{l}} c_{l}<0
$$

and

$$
S_{h}(E, C, C E L)=\frac{n_{l}}{n_{h}} \frac{E c_{l}}{n_{h} c_{h}+n_{l} c_{l}}>0
$$

Proof of Lemma 3. Below, we plug into $V_{j, i n}\left(P, n_{h, i n}, n_{l, i n}\right)=c_{j, i n} \frac{E}{\sum_{i \in N_{l} \cup N_{h}} c_{i, i n}}$,

$$
\begin{array}{rll}
\left(1+r_{f}\right) \sum_{i \in N_{l} \cup N_{h}} w_{i, i n} & \text { for } & E, \\
(1+r) \sum_{i \in N_{l} \cup N_{h}} w_{i, i n} & \text { for } & \sum_{i \in N_{l} \cup N_{h}} c_{i, i n}, \text { and } \\
(1+r) w_{j} & \text { for } & c_{j} .
\end{array}
$$

Hence,

$$
\begin{aligned}
V_{j, i n}\left(P, n_{h, i n}, n_{l, i n}\right) & =(1+r) w_{j} \frac{\left(1+r_{f}\right) \sum_{i \in N_{l} \cup N_{h}} w_{i, i n}}{(1+r) \sum_{i \in N_{l} \cup N_{h}} w_{i, i n}} \\
& =\left(1+r_{f}\right) w_{j}=P_{j}
\end{aligned}
$$

Proof of Lemma 4. (i) Pick any $n_{n, i n}>0$ and $n_{l, i n}>0$. We know by Corollary 2 that

$$
\begin{aligned}
& S_{l}\left(C E A, n_{h, i n}, n_{l, i n}\right)>0 \text { and } \\
& S_{h}\left(C E A, n_{h, i n}, n_{l, i n}\right)<0 .
\end{aligned}
$$

Therefore, by the definition of $c^{*}$ (see Lemma 1), $c_{l} \leq c^{*} \leq c_{h}$.

(ii) Pick any $n_{n, i n}>0$ and $n_{l, i n}>0$. We know by Corollary 2 that

$$
\begin{aligned}
S_{h}\left(C E L, n_{h, i n}, n_{l, i n}\right) & >0 \text { and } \\
S_{l}\left(C E L, n_{h, i n}, n_{l, i n}\right) & <0 .
\end{aligned}
$$

Therefore, by the definition of $\widetilde{c}$ (see Lemma 1), $c_{l} \leq \widetilde{c} \leq c_{h}$.

Proof of Lemma 5. (i) There are two possible cases for $C E A$ : (a-1) $c_{h}>c_{l}>\lambda_{\text {cea }}$ and $(\mathrm{a}-2) c_{h}>\lambda_{c e a} \geq c_{l}$. We know from Corollary 2 (a-1),

$$
S_{l}\left(C E A, n_{h, i n}, n_{l, i n}\right)=\frac{n_{h, i n}}{n_{h, i n}+n_{l, i n}}\left(1+r_{f}\right)\left(w_{h}-w_{l}\right) .
$$

Thus,

$$
\frac{\triangle S_{l}\left(C E A, n_{h, i n}, n_{l, i n}\right)}{\triangle n_{l, i n}}=-\frac{n_{h, i n}}{\left(n_{h, i n}+n_{l, i n}+1\right)\left(n_{h, i n}+n_{l, i n}\right)}\left(1+r_{f}\right)\left(w_{h}-w_{l}\right)<0 .
$$


On the other hand, in Corollary 2 (a-2),

$$
S_{l}\left(C E A, n_{h, i n}, n_{l, i n}\right)=\left(r-r_{f}\right) w_{l} .
$$

Thus,

$$
\frac{\triangle S_{l}\left(C E L, n_{h, i n}, n_{l, i n}\right)}{\triangle n_{l, i n}}=0
$$

(ii) There are two possible cases for $C E A$ : (a-1) and (a-2). We know from Corollary 2 (a-1),

$$
S_{h}\left(C E A, n_{h, i n}, n_{l, i n}\right)=\frac{n_{l, i n}}{n_{h, i n}+n_{l, i n}}\left(1+r_{f}\right)\left(w_{l}-w_{h}\right)
$$

Thus,

$$
\frac{\triangle S_{h}\left(C E A, n_{h, i n}, n_{l, i n}\right)}{\triangle n_{l, i n}}=\frac{n_{h, i n}\left(1+r_{f}\right)\left(w_{l}-w_{h}\right)}{\left(n_{h, i n}+n_{l, i n}+1\right)\left(n_{h, i n}+n_{l, i n}\right)}<0 .
$$

On the other hand, in Corollary 2 (a-2),

$$
S_{h}\left(C E A, n_{h, i n}, n_{l, i n}\right)=\frac{n_{l, i n}}{n_{h, i n}}\left(r_{f}-r\right) w_{l} .
$$

Thus,

$$
\frac{\triangle S_{h}\left(C E A, n_{h, i n}, n_{l, i n}\right)}{\triangle n_{l, i n}}=\frac{\left(r_{f}-r\right) w_{l}}{n_{h, i n}}<0 .
$$

(iii) There are two possible cases for CEL: (b-1) $c_{h}>c_{l}>\lambda_{c e l}$ and (b-2) $c_{h}>\lambda_{c e l} \geq$ $c_{l}$. We know from Corollary 2 (b-1),

$$
S_{l}\left(C E L, n_{h, i n}, n_{l, i n}\right)=\frac{n_{h, i n}}{n_{h, i n}+n_{l, i n}}\left(r-r_{f}\right)\left(w_{l}-w_{h}\right) .
$$

Thus,

$$
\frac{\triangle S_{l}\left(C E L, n_{h, i n}, n_{l, i n}\right)}{\triangle n_{h, i n}}=\frac{n_{l, i n}}{\left(n_{h, i n}+n_{l, i n}+1\right)\left(n_{h, i n}+n_{l, i n}\right)}\left(r-r_{f}\right)\left(w_{l}-w_{h}\right)<0 .
$$

On the other hand, in Corollary 2 (b-2),

$$
S_{l}\left(C E L, n_{h, i n}, n_{l, i n}\right)=-\left(1+r_{f}\right) w_{l} .
$$

Thus,

$$
\frac{\triangle S_{l}\left(C E L, n_{h, i n}, n_{l, i n}\right)}{\triangle n_{h, i n}}=0
$$

(iv) There are two possible cases for CEL: (b-1) and (b-2). We know from Corollary 2 (b-1),

$$
S_{h}\left(C E L, n_{h, i n}, n_{l, i n}\right)=\frac{n_{l, i n}}{n_{h, i n}+n_{l, i n}}\left[\left(r-r_{f}\right)\left(w_{h}-w_{l}\right)\right] .
$$

Thus,

$$
\frac{\triangle S_{h}\left(C E L, n_{h, i n}, n_{l, i n}\right)}{\triangle n_{h, i n}}=-\frac{n_{l, i n}}{\left(n_{h, i n}+n_{l, i n}+1\right)\left(n_{h, i n}+n_{l, i n}\right)}\left(r-r_{f}\right)\left(w_{h}-w_{l}\right)<0 .
$$


On the other hand, in Corollary 2 (b-2),

$$
S_{h}\left(C E L, n_{h, i n}, n_{l, i n}\right)=\frac{n_{l, i n}}{n_{h, i n}}\left(1+r_{f}\right) w_{l} .
$$

Thus,

$$
\frac{\triangle S_{h}\left(C E L, n_{h, i n}, n_{l, i n}\right)}{\triangle n_{h, i n}}=-\frac{n_{l, i n}}{\left(n_{h, i n}+1\right)\left(n_{h, i n}\right)}\left(1+r_{f}\right) w_{l}<0 .
$$

(v) There are two possible cases for $C E A$ : (a-1) $c_{h}>c_{l}>\lambda_{\text {cea }}$ and (a-2) $c_{h}>\lambda_{\text {cea }} \geq$ $c_{l}$. We know from Corollary 2 (a-1),

$$
S_{l}\left(C E A, n_{h, i n}, n_{l, i n}\right)=\frac{n_{h, i n}}{n_{h, i n}+n_{l, i n}}\left(1+r_{f}\right)\left(w_{h}-w_{l}\right) .
$$

Thus,

$$
\frac{\triangle S_{l}\left(C E A, n_{h, i n}, n_{l, i n}\right)}{\triangle n_{h, i n}}=\frac{n_{l, i n}}{\left(n_{h, i n}+n_{l, i n}+1\right)\left(n_{h, i n}+n_{l, i n}\right)}\left(1+r_{f}\right)\left(w_{h}-w_{l}\right)>0 .
$$

On the other hand, in Corollary 2 (a-2),

$$
S_{l}\left(C E A, n_{h, i n}, n_{l, i n}\right)=\left(r-r_{f}\right) w_{l} .
$$

Thus,

$$
\frac{\triangle S_{l}\left(C E L, n_{h, i n}, n_{l, i n}\right)}{\triangle n_{l, i n}}=0 .
$$

(vi) There are two possible cases for $C E A$ : (a-1) and (a-2). We know from Corollary $2(\mathrm{a}-1)$,

$$
S_{h}\left(C E A, n_{h, i n}, n_{l, i n}\right)=\frac{n_{l, i n}}{n_{h, i n}+n_{l, i n}}\left(1+r_{f}\right)\left(w_{l}-w_{h}\right)<0
$$

Thus,

$$
\frac{\triangle S_{h}\left(C E A, n_{h, i n}, n_{l, i n}\right)}{\triangle n_{h, i n}}=-\frac{n_{l, i n}}{\left(n_{h, \text { in }}+n_{l, i n}+1\right)\left(n_{h, \text { in }}+n_{l, i n}\right)}\left(1+r_{f}\right)\left(w_{l}-w_{h}\right)>0 .
$$

On the other hand, in Corollary 2 (a-2),

$$
S_{h}\left(C E A, n_{h, i n}, n_{l, i n}\right)=\frac{n_{l, i n}}{n_{h, i n}}\left(r_{f}-r\right) w_{l}<0 .
$$

Thus,

$$
\frac{\triangle S_{h}\left(C E A, n_{h, i n}, n_{l, i n}\right)}{\triangle n_{h, i n}}=-\frac{n_{l, i n}}{n_{h, i n}\left(n_{h, i n}+1\right)}\left(r_{f}-r\right) w_{l}>0 .
$$

(vii) There are two possible cases for CEL: (b-1) $c_{h}>c_{l}>\lambda_{c e l}$ and (b-2) $c_{h}>$ $\lambda_{\text {cel }} \geq c_{l}$. We know from Corollary 2 (b-1),

$$
S_{l}\left(C E L, n_{h, i n}, n_{l, i n}\right)=\frac{n_{h, i n}}{n_{h, i n}+n_{l, i n}}\left(r-r_{f}\right)\left(w_{l}-w_{h}\right)<0 .
$$


Thus,

$$
\frac{\triangle S_{l}\left(C E L, n_{h, i n}, n_{l, i n}\right)}{\triangle n_{l, i n}}=-\frac{n_{h, i n}}{\left(n_{h, i n}+n_{l, i n}+1\right)\left(n_{h, i n}+n_{l, i n}\right)}\left(r-r_{f}\right)\left(w_{l}-w_{h}\right)>0 .
$$

On the other hand, in Corollary 2 (b-2),

$$
S_{l}\left(C E L, n_{h, i n}, n_{l, i n}\right)=-\left(1+r_{f}\right) w_{l}<0 .
$$

Therefore,

$$
\frac{\triangle S_{l}\left(C E L, n_{h, i n}, n_{l, i n}\right)}{\triangle n_{l, i n}}=0
$$

(viii) There are two possible cases for $C E L$ : (b-1) and (b-2). We know from Corollary $2(\mathrm{~b}-1)$,

$$
S_{h}\left(C E L, n_{h, i n}, n_{l, i n}\right)=\frac{n_{l, i n}}{n_{h, i n}+n_{l, i n}}\left[\left(r-r_{f}\right)\left(w_{h}-w_{l}\right)\right]>0 .
$$

Thus,

$$
\frac{\triangle S_{h}\left(C E L, n_{h, i n}, n_{l, i n}\right)}{\triangle n_{l, i n}}=\frac{n_{h, i n}}{\left(n_{h, i n}+n_{l, i n}+1\right)\left(n_{h, i n}+n_{l, i n}\right)}\left[\left(r-r_{f}\right)\left(w_{h}-w_{l}\right)\right]>0 .
$$

On the other hand, in Corollary 2 (b-2),

$$
S_{h}\left(C E L, n_{h, i n}, n_{l, i n}\right)=\frac{n_{l, i n}}{n_{h, i n}}\left(1+r_{f}\right) w_{l} .
$$

Thus,

$$
\frac{\triangle S_{h}\left(C E L, n_{h, i n}, n_{l, i n}\right)}{\triangle n_{l, i n}}=\frac{\left(n_{l, i n}+1\right)}{n_{h, i n}}\left(1+r_{f}\right) w_{l}>0
$$

Proof of Lemma 6. We know that $P_{t}=\left(1+r_{f}\right) w_{t}$ and $V_{t}=(1+\bar{r}) w_{t}$. The expected payoff of a type $t$ agent under $P$ can be written as

$$
P_{i}^{e}=\pi_{s}(1+r) w_{t}+\left(1-\pi_{s}\right)\left(1+r_{f}\right) w_{t} .
$$

Therefore, a type $t$ agent chooses to play in under $P$ if

$$
P_{i}^{e}=\pi_{s}(1+r) w_{t}+\left(1-\pi_{s}\right)\left(1+r_{f}\right) w_{t} \geq(1+\bar{r}) w_{t}=V_{i, \text { out }} .
$$

This condition can be rewritten as

$$
\pi_{s}(1+r)+\left(1-\pi_{s}\right)\left(1+r_{f}\right) \geq(1+\bar{r}) .
$$

Since there are no income parameters in this inequality, it implies that for any two agents of different types, the condition determining their strategies under $P$ is identical, i.e.,

$$
P_{h}^{e} \geq V_{h, \text { out }} \Leftrightarrow P_{l}^{e} \geq V_{l, \text { out }} .
$$


Proof of Lemma 7. We analyze $P, C E A$, and $C E L$ separately since the argumentation for each case will be slightly different.

(P) Suppose, by a contradiction, that two agents $i$ and $j$ of type $t$ play different strategies in equilibrium. By Lemma $3, P_{i}=\left(1+r_{f}\right) w_{i}$ in case of bankruptcy. On the other hand, agent $i$ 's payoff is $(1+r) w_{i}$ in case of successful completion of the project and the outside option pays $(1+\bar{r}) w_{i}$. Since for all $i, j \in N_{t}$,

$$
\begin{aligned}
& P_{i}^{e}=\pi_{s}\left[(1+r) w_{t}\right]+\left(1-\pi_{s}\right)\left[\left(1+r_{f}\right) w_{t}\right] \text { and } \\
& P_{j}^{e}=\pi_{s}\left[(1+r) w_{t}\right]+\left(1-\pi_{s}\right)\left[\left(1+r_{f}\right) w_{t}\right]
\end{aligned}
$$

we have

$$
\begin{aligned}
& \pi_{s}\left[(1+r) w_{t}\right]+\left(1-\pi_{s}\right)\left[\left(1+r_{f}\right) w_{t}\right] \geq(1+\bar{r}) w_{t} \Leftrightarrow \\
& \pi_{s}\left[(1+r) w_{t}\right]+\left(1-\pi_{s}\right)\left[\left(1+r_{f}\right) w_{t}\right] \geq(1+\bar{r}) w_{t} .
\end{aligned}
$$

However, this logical statement implies that agents $i$ and $j$ cannot have different strategies in equilibrium; a contradiction. Hence the result follows.

The proofs for $C E A$ and $C E L$ require a separate analysis for $l$ type agents and $h$ type agents, since these agents are treated differently by $C E A$ and $C E L$.

(CEA) Suppose, by contradiction, that in equilibrium there exists an agent $i \in N_{h}$ who plays in whereas another agent $j \in N_{h}$ plays out under $C E A$. We denote the number of type $h$ agents playing in as $n_{h, i n}$ and the number of type $l$ agents playing in as $n_{l, i n}$. We consider two cases: (CEA - i) there is at least one agent $k \in N_{l}$ who plays in and (CEA - ii) there is no agent $k \in N_{l}$ who plays $i n$.

(CEA - i) If there is at least one agent $k \in N_{l}$ who plays $i n$, i.e., $n_{l, i n} \geq 1$, by Corollary 2,

$$
\begin{aligned}
& \text { if } c_{h}>c_{l}>\lambda_{c e a}, \text { then } S_{h}(C E A, i n)=\frac{n_{l}}{n_{h}+n_{l}}\left[\left(1+r_{f}\right)\left(w_{l}-w_{h}\right)\right]<0 \text { and } \\
& \text { if } c_{h}>\lambda_{c e a} \geq c_{l}, \text { then } S_{h}(C E A, i n)=\frac{n_{l}\left(r_{f}-r\right) w_{l}}{n_{h}}<0 .
\end{aligned}
$$

Hence, we know that $S_{i}\left(C E A, n_{h, i n}, n_{l, i n}\right)<0$. Also, the equilibrium property and our tie-breaking assumption imply that

$$
V_{i, i n}^{e}\left(C E A, n_{h, i n}, n_{l, i n}\right)=P_{i}^{e}+\left(1-\pi_{s}\right) S_{i}\left(C E A, n_{h, i n}, n_{l, i n}\right) \geq V_{i, o u t}
$$

and

$$
V_{j, \text { in }}^{e}\left(C E A, n_{h, \text { in }}+1, n_{l, \text { in }}\right)=P_{j}^{e}+\left(1-\pi_{s}\right) S_{j}\left(C E A, n_{h, \text { in }}+1, n_{l, \text { in }}\right)<V_{j, \text { out }} .
$$

Now, assume that player $j$ switches to $i n$. Then, number of type $h$ agents playing in is $n_{h, i n}+1$. The definition of $C E A$, the equilibrium property, and our tie-breaking assumption imply that

$$
V_{i, i n}^{e}\left(C E A, n_{h, \text { in }}+1, n_{l, i n}\right)=V_{j, i n}^{e}\left(C E A, n_{h, \text { in }}+1, n_{l, i n}\right)<V_{j, \text { out }} \equiv V_{i, \text { out }} .
$$


On the other hand, by Corollary 2 and Lemma 5 ,

$$
S_{i}\left(C E A, n_{h, i n}, n_{l, i n}\right)<S_{i}\left(C E A, n_{h, i n}+1, n_{l, i n}\right)<0 .
$$

Hence,

$$
V_{i, i n}^{e}\left(C E A, n_{h, i n}, n_{l, i n}\right)<V_{i, i n}^{e}\left(C E A, n_{h, i n}+1, n_{l, i n}\right) .
$$

Therefore,

$$
\begin{aligned}
V_{i, \text { in }}^{e}\left(C E A, n_{h, \text { in }}, n_{l, i n}\right) & <V_{i, \text { in }}^{e}\left(C E A, n_{h, \text { in }}+1, n_{l, \text { in }}\right) \leq V_{i, \text { out }} \\
\Rightarrow V_{i, \text { in }}^{e}\left(C E A, n_{h, \text { in }}, n_{l, i n}\right) & <V_{i, \text { out }} .
\end{aligned}
$$

However, if the above inequality is satisfied, agent $i$ playing in at the beginning cannot be an optimal action for him; a contradiction. Hence the result follows.

(CEA - ii) If there is no agent $k \in N_{l}$ who plays in under $C E A$ in equilibrium, i.e., $n_{l, i n}=0$, this implies

$$
\begin{aligned}
S_{i}\left(C E A, n_{h, i n}, n_{l, i n}\right) & =0 \text { and } \\
V_{i, i n}^{e}\left(C E A, n_{h, i n}, n_{l, i n}\right) & =P_{i}^{e} .
\end{aligned}
$$

However, this implies that $P_{i}^{e} \geq V_{i, \text { out }}$. Hence, from (P) the result follows.

Now, suppose that in equilibrium there exists an agent $i \in N_{l}$ who plays in whereas another agent $j \in N_{l}$ plays out under $C E A$. We consider two cases: (CEA iii) there is at least one agent $k \in N_{h}$ who plays in and (CEA - iv) there is no agent $k \in N_{h}$ who plays in.

(CEA - iii) If there is at least one agent $k \in N_{h}$ who plays $i n$, i.e., $n_{h, i n} \geq 1$, by Corollary 2,

$$
\begin{aligned}
& \text { if } c_{h}>c_{l}>\lambda_{\text {cea }}, \text { then } S_{h}(C E A, i n)=\frac{n_{l}}{n_{h}+n_{l}}\left[\left(1+r_{f}\right)\left(w_{l}-w_{h}\right)\right]<0 \text { and } \\
& \text { if } c_{h}>\lambda_{c e a} \geq c_{l}, \text { then } S_{h}(C E A, i n)=\frac{n_{l}\left(r_{f}-r\right) w_{l}}{n_{h}}<0 .
\end{aligned}
$$

Hence, we know that

$$
\begin{aligned}
S_{k}\left(C E A, n_{h, i n}, n_{l, i n}\right) & <0 \text { and } \\
V_{k, \text { in }}^{e}\left(C E A, n_{h, \text { in }}, n_{l, i n}\right) & =P_{k}^{e}+\left(1-\pi_{s}\right) S_{k}\left(C E A, n_{h, \text { in }}, n_{l, \text { in }}\right) \geq V_{k, \text { out }} .
\end{aligned}
$$

Now, assume that agent $j$ switches to playing in. The definition of $C E A$, the equilibrium property, and our tie-breaking assumption imply that

$$
V_{i, \text { in }}^{e}\left(C E A, n_{h, \text { in }}, n_{l, \text { in }}+1\right)=V_{j, \text { in }}^{e}\left(C E A, n_{h, \text { in }}, n_{l, \text { in }}+1\right)<V_{j, \text { out }} \equiv V_{i, \text { out }}
$$

where,

$$
\begin{aligned}
V_{j, i n}^{e}\left(C E A, n_{h, i n}, n_{l, i n}+1\right) & =P_{j}^{e}+\left(1-\pi_{s}\right) S_{j}\left(C E A, n_{h, i n}, n_{l, i n}+1\right) \text { and } \\
S_{j}\left(C E A, n_{h, i n}, n_{l, i n}+1\right) & >0 .
\end{aligned}
$$


However, if

$$
V_{k, \text { in }}^{e}\left(C E A, n_{h, \text { in }}, n_{l, i n}\right)=P_{k}^{e}+\left(1-\pi_{s}\right) S_{k}\left(C E A, n_{h, \text { in }}, n_{l, i n}\right) \geq V_{k, \text { out }},
$$

then by proportionality,

$$
P_{k}^{e} \geq V_{k, \text { out }} \Leftrightarrow P_{j}^{e} \geq V_{j, \text { out }}
$$

and by Corollary 2 and Lemma 5

$$
S_{k}\left(C E A, n_{h, i n}, n_{l, i n}\right)<0 \text { and } S_{j}\left(C E A, n_{h, i n}, n_{l, i n}+1\right)>0
$$

leads to

$$
V_{j, i n}^{e}\left(C E A, n_{h, i n}, n_{l, i n}+1\right)=V_{i, i n}^{e}\left(C E A, n_{h, i n}, n_{l, i n}+1\right) \leq V_{j, \text { out }},
$$

which is a contradiction. Thus, in equilibrium if it is an optimal action for a type $h$ agent to play in under $C E A$, it cannot be an optimal action for any type $l$ agent to play out, since any type $l$ agent will have non-negative transfers. Hence, the result follows.

(CEA - iv) If there is no agent $k \in N_{h}$ who plays in under $C E A$ in equilibrium, i.e., $n_{h, i n}=0$, this implies

$$
\begin{aligned}
S_{i}\left(C E A, n_{h, i n}, n_{l, i n}\right) & =0 \text { and } \\
V_{i, i n}^{e}\left(C E A, n_{h, i n}, n_{l, i n}\right) & =P_{i}^{e} .
\end{aligned}
$$

Then, if agent $j$ switches to playing $i n$, by Corollary 2 and Lemma 5 ,

$$
S_{k}\left(C E A, n_{h, i n}, n_{l, i n}\right)>S_{k}\left(C E A, n_{h, i n}, n_{l, i n}+1\right) .
$$

Then, there will still be no agent $k \in N_{h}$ who plays $i n$. Hence, expected payoffs of type $l$ agents will not be affected by the existence of other type $l$ agents playing $i n$, which implies

$$
\begin{aligned}
V_{i, i n}^{e}\left(C E A, n_{h, i n}, n_{l, i n}\right) & =V_{i, i n}^{e}\left(C E A, n_{h, i n}, n_{l, i n}+1\right) \\
& =V_{j, \text { in }}^{e}\left(C E A, n_{h, i n}, n_{l, i n}+1\right) \\
\Rightarrow V_{i, \text { in }}^{e}\left(C E A, n_{h, i n}, n_{l, i n}\right) & <V_{i, \text { out }} .
\end{aligned}
$$

If the above inequality is satisfied, playing in at the beginning cannot be an optimal action for agent $i$; a contradiction. Hence the result follows.

(CEL) The proof is similar to the proof for $C E A$. Suppose, by contradiction, that in equilibrium there exists an agent $i \in N_{l}$ who plays in whereas another agent $j \in N_{l}$ plays out under $C E L$. We denote the number of type $h$ agents playing in as $n_{h, i n}$ and the number of type $l$ agents playing in as $n_{l, i n}$. We consider two cases: (CEL i) there is at least one agent $k \in N_{h}$ who plays in (CEL - ii) there is no agent $k \in N_{h}$ who plays $i n$.

(CEL - i) If there is at least one agent $k \in N_{h}$ who plays in, Corollary 2, the equilibrium property and our tie-breaking assumption imply that

$$
V_{i, \text { out }} \leq V_{i, i n}^{e}\left(C E L, n_{h, i n}, n_{l, i n}\right) \text { and }
$$




$$
V_{j, i n}^{e}\left(C E L, n_{h, \text { in }}+1, n_{l, i n}\right)<V_{j, \text { out }} .
$$

Now assume that player $j$ switches to $i n$. The number of type $l$ agents playing in is $n_{l}+1$ now. The definition of $C E L$, the equilibrium property, and our tie-breaking assumption imply that

$$
V_{i, i n}^{e}\left(C E L, n_{h, i n}, n_{l, i n}+1\right)=V_{j, i n}^{e}\left(C E L, n_{h, \text { in }}, n_{l, \text { in }}+1\right)<V_{j, \text { out }} \equiv V_{i, \text { out }} .
$$

On the other hand, by Lemma 5,

$$
S_{i}\left(C E L, n_{h, i n}, n_{l, i n}+1\right)>S_{i}\left(C E L, n_{h, i n}, n_{l, i n}\right) .
$$

Hence,

$$
V_{i, i n}^{e}\left(C E L, n_{h, i n}, n_{l, i n}+1\right)>V_{i, i n}^{e}\left(C E L, n_{h, i n}, n_{l, i n}\right) .
$$

Therefore,

$$
\begin{aligned}
V_{i, \text { in }}^{e}\left(C E L, n_{h, \text { in }}, n_{l, i n}\right) & <V_{i, \text { in }}^{e}\left(C E L, n_{h, \text { in }}, n_{l, i n}+1\right) \leq V_{i, \text { out }} \\
\Rightarrow V_{i, \text { in }}^{e}\left(C E L, n_{h, \text { in }}, n_{l, \text { in }}\right) & <V_{i, \text { out }} .
\end{aligned}
$$

However, if the above inequality is satisfied playing in at the beginning cannot be an optimal action for agent $i$; a contradiction. Hence the result follows.

(CEL - ii) If there is no agent $k \in N_{l}$ who plays in under $C E L$ in equilibrium (i.e., $\left.n_{l, i n}=0\right)$, this implies

$$
\begin{aligned}
S_{i}\left(C E L, n_{h, i n}, n_{l, i n}\right) & =0 \text { and } \\
V_{i, i n}^{e}\left(C E L, n_{h, i n}, n_{l, i n}\right) & =P_{i}^{e} .
\end{aligned}
$$

However, this implies that $P_{i}^{e} \geq V_{i, \text { out }}$. Hence, from (P) the result follows.

Now, suppose, by contradiction, that in equilibrium there exists an agent $i \in N_{h}$ who plays $i n$, whereas another agent $j \in N_{h}$ plays out. We consider two cases: (CEL - iii) there is at least one agent $k \in N_{l}$ who plays in (CEL - iv) there is no agent $k \in N_{l}$ who plays $i n$.

(CEL - iii) If there is at least one agent $k \in N_{l}$ who plays $i n$, by Corollary 2,

$$
\begin{aligned}
& \text { if } c_{h}>c_{l}>\lambda_{c e l}, S_{l}(C E L, i n)=\frac{n_{h}}{n_{h}+n_{l}}\left(r-r_{f}\right)\left(w_{l}-w_{h}\right) \text { and } \\
& \text { if } c_{h}>\lambda_{c e l} \geq c_{l}, S_{l}(C E L, i n)=-\left(1+r_{f}\right) w_{l} .
\end{aligned}
$$

Hence, we know that

$$
\begin{aligned}
S_{k}\left(C E L, n_{h, i n}, n_{l, i n}\right) & <0 \text { and } \\
V_{k, \text { in }}^{e}\left(C E L, n_{h, \text { in }}, n_{l, i n}\right) & =P_{k}^{e}+S_{k}\left(C E L, n_{h, \text { in }}, n_{l, i n}\right) \geq V_{k, \text { out }} .
\end{aligned}
$$

Now, assume that agent $j$ switches to playing $i n$. The definition of $C E L$, the equilibrium property and our tie-breaking assumption imply that

$$
V_{j, \text { in }}^{e}\left(C E L, n_{h, \text { in }}+1, n_{l, \text { in }}\right)=V_{i, \text { in }}^{e}\left(C E L, n_{h, \text { in }}+1, n_{l, \text { in }}\right) \leq V_{i, \text { out }} \equiv V_{j, \text { out }}
$$


where,

$$
\begin{aligned}
V_{j, i n}^{e}\left(C E L, n_{h, i n}+1, n_{l, i n}\right) & =P_{j}^{e}+\left(1-\pi_{s}\right) S_{j}\left(C E L, n_{h, i n}+1, n_{l, i n}\right) \text { and } \\
S_{j}\left(C E L, n_{h, i n}+1, n_{l, i n}\right) & >0 .
\end{aligned}
$$

However, if

$$
V_{k, \text { in }}^{e}\left(C E L, n_{h, \text { in }}, n_{l, i n}\right)=P_{k}^{e}+\left(1-\pi_{s}\right) S_{k}\left(C E L, n_{h, \text { in }}, n_{l, \text { in }}\right) \geq V_{k, \text { out }}
$$

then by proportionality,

$$
P_{k}^{e} \geq V_{k, \text { out }} \Leftrightarrow P_{j}^{e} \geq V_{j, \text { out }}
$$

and by Corollary 2 and Lemma 5

$$
S_{k}\left(C E L, n_{h, i n}, n_{l, i n}\right)<0 \text { and } S_{j}\left(C E L, n_{h, i n}, n_{l, i n}+1\right)>0
$$

leads to

$$
V_{j, \text { in }}^{e}\left(C E L, n_{h, i n}, n_{l, i n}+1\right)=V_{i, i n}^{e}\left(C E L, n_{h, \text { in }}, n_{l, \text { in }}+1\right) \leq V_{j, \text { out }},
$$

which is a contradiction. Thus, if it is optimal for a type $l$ agent to play in under $C E L$ in equilibrium, it cannot be optimal for any type $h$ agent to play out in equilibrium, since any type $h$ agent will have non-negative transfers. Hence, the result follows.

(CEL - iv) If there is no agent $k \in N_{l}$ who plays in under $C E L$ in equilibrium (i.e., $n_{l, \text { in }}=0$ ), this implies

$$
\begin{aligned}
S_{i}\left(C E L, n_{h, i n}, n_{l, i n}\right) & =0 \text { and } \\
V_{i, i n}^{e}\left(C E L, n_{h, i n}, n_{l, i n}\right) & =P_{i, i n}^{e} .
\end{aligned}
$$

Then if agent $j$ switches to playing in, by Lemma 5 ,

$$
S_{k}\left(C E L, n_{h, i n}, n_{l, i n}\right)>S_{k}\left(C E L, n_{h, i n}+1, n_{l, i n}\right) .
$$

Then, there will still be no agent $k \in N_{l}$ who plays $i n$.Hence, the expected payoffs of type $h$ agents will not be affected by the existence of other type $h$ agents playing in, which implies

$$
\begin{aligned}
V_{i, \text { in }}^{e}\left(C E L, n_{h, \text { in }}, n_{l, i n}\right) & =V_{j, \text { in }}^{e}\left(C E L, n_{h, \text { in }}+1, n_{l, i n}\right) \\
& =V_{i, \text { in }}^{e}\left(C E L, n_{h, \text { in }}+1, n_{l, \text { in }}\right) \\
& \leq V_{j, \text { out }} \equiv V_{i, \text { out }} .
\end{aligned}
$$

If the inequality above is satisfied strictly, then playing in at the beginning cannot be an optimal action for agent $i$; a contradiction. If it is satisfied, on the other hand, with equality, then it is not optimal for agent $j$ to play out at the beginning (due to the tie-breaking rule) which is again a contradiction. Hence the result follows.

Proof of Lemma 8. (i) By Corollary 2, $S_{l}(C E A$, in $)>0$ and $S_{h}(C E A$, in $)<0$. Therefore, if type $l$ agents (who are favored by $C E A$ ) play out, this implies that

$$
V_{l}^{e}(C E A, \text { in })=P_{l}^{e}+\left(1-\pi_{s}\right) S_{l}(C E A, \text { in })<V_{l, \text { out }} .
$$


Since $S_{l}(C E A$, in $)>0$, this implies that $P_{l}^{e}<V_{l, \text { out }}$. Using proportionality, $P_{l}^{e}<$ $V_{l, \text { out }}$ if and only if $P_{h}^{e}<V_{h, \text { out }}$. However, we know that $S_{h}(C E A, i n)<0$. Therefore,

$$
V_{h}^{e}(C E A, \text { in })=P_{h}^{e}+\left(1-\pi_{s}\right) S_{h}(C E A, \text { in })>V_{h, \text { out }}
$$

cannot hold. Hence, this implies that playing in cannot be optimal for type $h$ agents (who are disfavored by $C E A$ ), if it is not optimal for type $l$ agents.

(ii) By Corollary 2, $S_{h}(C E L, i n)>0$ and $S_{l}(C E L, i n)<0$. Therefore, if type $h$ agents (who are favored by $C E L$ ) play out, this implies that

$$
V_{h}^{e}(C E L, \text { in })=P_{h}^{e}+\left(1-\pi_{s}\right) S_{h}(C E L, \text { in })<V_{h, \text { out }} .
$$

Since $S_{h}(C E L$, in $)>0$, this implies that $P_{h}^{e}<V_{h, \text { out }}$. Using proportionality, $P_{h}^{e}<$ $V_{h, \text { out }}$ if and only if $P_{l}^{e}<V_{l, \text { out }}$. However, we know that $S_{l}(C E L$, in $)<0$. Therefore,

$$
V_{l}^{e}(C E L, \text { in })=P_{l}^{e}+\left(1-\pi_{s}\right) S_{l}(C E L, \text { in })>V_{l, \text { out }}
$$

cannot hold. Hence, this implies that playing in cannot be optimal for type $l$ agents (who are disfavored by $C E L$ ), if it is not optimal for type $h$ agents.

The comparative statics in the following appendix sections are used in obtaining the results in Section 5 .

\section{C - Comparative static analyses on $n_{l}, w_{l}, n_{h}$ and $w_{h}$}

\begin{tabular}{|c|c|c|c|c|}
\hline & \multicolumn{2}{|c|}{$S_{h}^{c e a}$} & \multicolumn{2}{c|}{$S_{l}^{c e a}$} \\
\hline & $c_{h}>\lambda_{c e a} \geq c_{l}$ & $c_{h}>c_{l} \geq \lambda_{c e a}$ & $c_{h}>\lambda_{\text {cea }} \geq c_{l}$ & $c_{h}>c_{l} \geq \lambda_{c e a}$ \\
\hline$n_{l}$ & - & - & 0 & - \\
\hline$n_{h}$ & + & + & 0 & + \\
\hline$w_{l}$ & - & + & + & - \\
\hline$w_{h}$ & 0 & - & 0 & + \\
\hline
\end{tabular}

Table C1: The Effect of Changes in $n_{l}, n_{h}, w_{l}$ and $w_{h}$ on $S_{t}^{c e a}$

\begin{tabular}{|c|c|c|c|c|}
\hline & \multicolumn{2}{|c|}{$S_{h}^{c e l}$} & \multicolumn{2}{c|}{$S_{l}^{c e l}$} \\
\hline & $c_{h}>c_{l}>\lambda_{c e l}$ & $c_{h}>\lambda_{c e l} \geq c_{l}$ & $c_{h}>c_{l}>\lambda_{c e l}$ & $c_{h}>\lambda_{c e l} \geq c_{l}$ \\
\hline$n_{l}$ & + & + & + & 0 \\
\hline$n_{h}$ & - & - & - & 0 \\
\hline$w_{l}$ & - & + & + & - \\
\hline$w_{h}$ & + & 0 & - & 0 \\
\hline
\end{tabular}

Table C2: The Effect of Changes in $n_{l}, n_{h}, w_{l}$ and $w_{h}$ on $S_{t}^{c e l}$

\section{D - Comparative static analyses on $r$ and $r_{f}$}

\begin{tabular}{|c|c|c|c|c|}
\hline & \multicolumn{2}{|c|}{$S_{h}^{\text {cea }}$} & \multicolumn{2}{c|}{$S_{l}^{\text {cea }}$} \\
\hline & $c_{h}>\lambda_{\text {cea }} \geq c_{l}$ & $c_{h}>c_{l} \geq \lambda_{\text {cea }}$ & $c_{h}>\lambda_{\text {cea }} \geq c_{l}$ & $c_{h}>c_{l} \geq \lambda_{c e a}$ \\
\hline$r$ & - & 0 & + & 0 \\
\hline$r_{f}$ & + & - & - & + \\
\hline
\end{tabular}


Table D1: The Effect of Changes in $r$ and $r_{f}$ on $S_{t}^{c e a}$

\begin{tabular}{|c|c|c|c|c|}
\hline & \multicolumn{2}{|c|}{$S_{h}^{c e l}$} & \multicolumn{2}{c|}{$S_{l}^{\text {cel }}$} \\
\hline & $c_{h}>c_{l}>\lambda_{c e l}$ & $c_{h}>\lambda_{\text {cel }} \geq c_{l}$ & $c_{h}>c_{l}>\lambda_{c e l}$ & $c_{h}>\lambda_{c e l} \geq c_{l}$ \\
\hline$r$ & + & 0 & - & 0 \\
\hline$r_{f}$ & - & + & + & - \\
\hline
\end{tabular}

Table D2: The Effect of Changes in $r$ and $r_{f}$ on $S_{t}^{c e l}$ 